\title{
Chiral approximation to twisted bilayer graphene: Exact intravalley inversion symmetry, nodal structure, and implications for higher magic angles
}

\author{
Jie Wang $\odot,{ }^{1, *}$ Yunqin Zheng, ${ }^{2,3}$ Andrew J. Millis ${ }^{1,4, \dagger}$ and Jennifer Cano $\oplus^{1,5, \$}$ \\ ${ }^{1}$ Center for Computational Quantum Physics, Flatiron Institute, 162 th Avenue, New York, New York 10010, USA \\ ${ }^{2}$ Institute for Solid State Physics, University of Tokyo, Kashiwa, Chiba 277-8581, Japan \\ ${ }^{3}$ Kavli Institute for the Physics and Mathematics of the Universe, University of Tokyo, Kashiwa, Chiba 277-8583, Japan \\ ${ }^{4}$ Department of Physics, Columbia University, 538 W 120th Street, New York, New York 10027, USA \\ ${ }^{5}$ Department of Physics and Astronomy, Stony Brook University, Stony Brook, New York 11974, USA
}

(Received 21 October 2020; accepted 17 February 2021; published 28 May 2021)

\begin{abstract}
This paper presents a mathematical and numerical analysis of the flat-band wave functions occurring in the chiral model of twisted bilayer graphene at the "magic" twist angles. We show that the chiral model possesses an exact intravalley inversion symmetry. Writing the flat-band wave function as a product of a lowest Landau level quantum Hall state and a spinor, we show that the components of the spinor are antiquantum Hall wave functions related by the inversion symmetry operation introduced here. We then show numerically that as one moves from the lowest to higher magic angles, the spinor components of the wave function exhibit an increasing number of zeros, resembling the changes in the quantum Hall wave function as the Landau level index is increased. The wave function zeros are characterized by a chirality, with zeros of the same chirality clustering near the center of the moiré unit cell, while opposite chirality zeros are pushed to the boundaries of the unit cell. The enhanced phase winding at higher magic angles suggests an increased circulating current. Physical implications for scanning tunneling spectroscopy, orbital magnetization and interaction effects are discussed.
\end{abstract}

DOI: 10.1103/PhysRevResearch.3.023155

\section{INTRODUCTION}

When one graphene layer is stacked on top of another layer with small relative twist angle, a moire super-lattice pattern is created. At particular twist angles, referred to by Bistritzer and MacDonald as "magic angles" [1], the bands near the chemical potential are dramatically flattened and separated from other bands [1-4]. Experiments on "magic angle" bilayers report interesting phenomena including superconductivity, interaction-driven insulating states and anomalous Hall effects [5-29].

There are eight flat bands arising from the combinations of degrees of freedom in the conduction bands of the component graphene layers [30-47]. The states that comprise these bands may be labeled by a spin degree of freedom and two additional indices labeling the layer and sublattice of the component graphene sheets. Much of the novel physics of twisted bilayer graphene is believed to arise when the symmetries corresponding to these quantum numbers are spontaneously or explicitly broken. Interestingly, many of the broken symmetry states appear to have a topological character, revealed

\footnotetext{
*jiewang@ flatironinstitute.org

†amillis@flatironinstitute.org

¥jcano-affiliate@ flatironinstitute.org
}

Published by the American Physical Society under the terms of the Creative Commons Attribution 4.0 International license. Further distribution of this work must maintain attribution to the author(s) and the published article's title, journal citation, and DOI. for example by anomalous Hall effects [7,8,41,48-51], and at least at integer filling, the topological character is believed to be inherent in the single-particle wave functions. An improved understanding of the single-particle wave functions is therefore important both for improved understandings of the observed and potentially observable topological phases and as a basis for theories of interaction effects in magic angle bilayer graphene.

Recently, Tarnopolsky, Kruchkov, and Vishwanath [52] drew attention to a particular "chiral" model in which the interlayer tunneling Hamiltonian contains no terms in which an electron hops from one layer to the same sublattice on the other layer. They showed that in this case the eight weakly dispersing bands become exactly flat (dispersionless) at certain twist angles. They further constructed explicit expressions for the zero-mode wave functions, and noticed that their solutions exhibited a holomorphic character reminiscent of the lowest Landau level quantum Hall physics [52,53]. This holomorphic character can give rise to a nontrivial topology of the flat bands, explaining the anomalous Hall effects.

In this paper, we study the zero-mode wave functions of the chiral model [52] of twisted bilayer graphene in more detail. We identify an exact intravalley inversion symmetry of the chiral model and show how this symmetry implies that the flat-band wave functions found by Tarpolsky, Kruchkov, and Vishwanath can be written (up to a normalization factor) as

$$
\phi_{k}(\boldsymbol{r})=\left(\begin{array}{c}
i \mathcal{G}(\boldsymbol{r}) \\
\eta \mathcal{G}(-\boldsymbol{r})
\end{array}\right) \times \Phi_{k}(\boldsymbol{r})
$$


Bottom Component

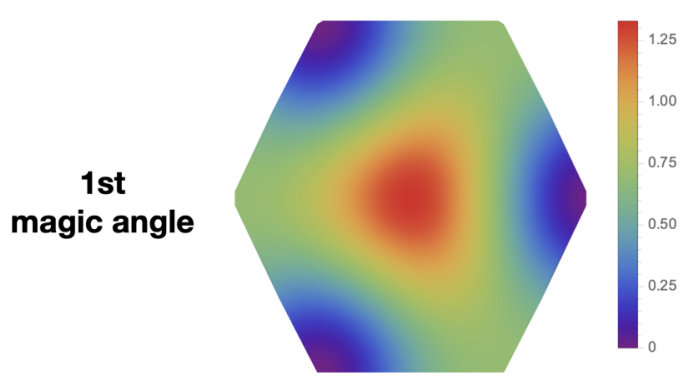

2nd magic angle

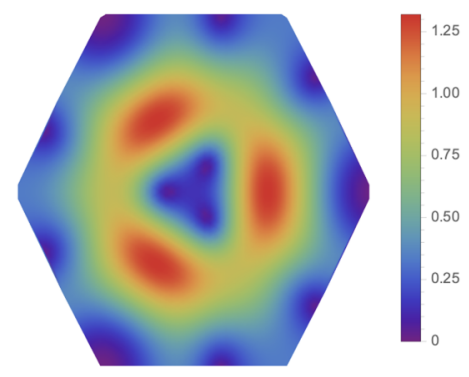

3rd magic angle
Top Component
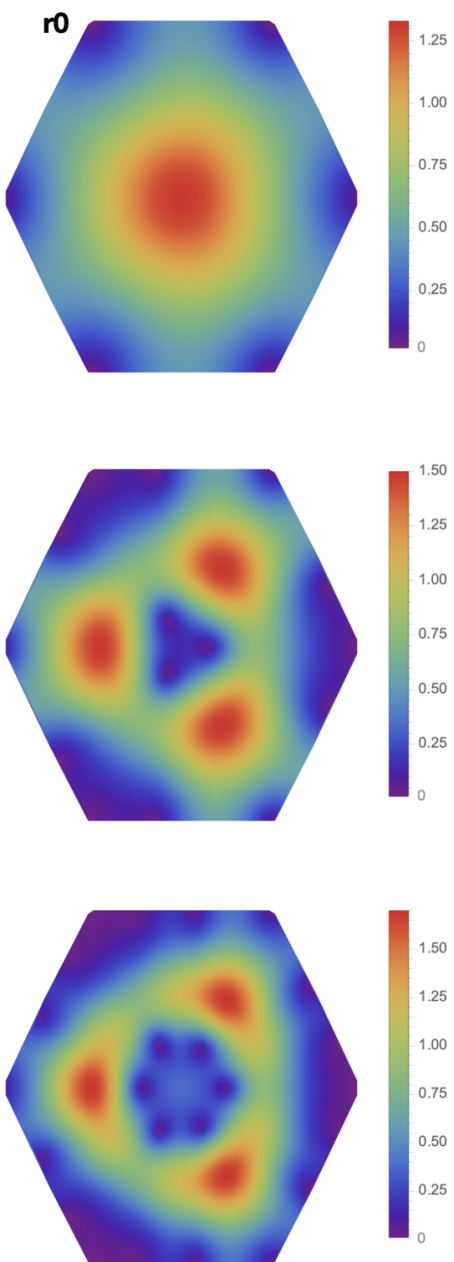

FIG. 1. Norm of each component of the wave function Eq. (1) at the moire Dirac point $\boldsymbol{K}$, plotted at the first three magic angles (three rows). The upper left and lower right corners of the unit cells are the $B A\left(\boldsymbol{r}_{0}\right)$ and $A B\left(-\boldsymbol{r}_{0}\right)$ stacking points, as marked. The two columns correspond to the bottom and top components of the wave function. Each of them has clear symmetry and zero structures. The wave functions at other Bloch momentum have a similar zero structure, as explained in the text. The zeros are classified by their chirality, i.e., whether the wave-function phase advances by $\pm 2 \pi$ when encircling the zero once. Remarkably, the wave function associated with the $n_{t h}$ magic angle has $3(n-1)$ zeros located at the unit cell center, all of which have the same chirality. We discuss the mathematical structure in Secs. IV and V, and implications for experimental observables in Sec. VI.

where $\Phi_{k}$ is a quantum Hall wave function of the lowest Landau level, the function $\mathcal{G}(\boldsymbol{r})$ can be interpreted as a quantum Hall wave function in a magnetic field oppositely directed to that of $\Phi_{k}$, and $\eta= \pm 1$ is the inversion eigenvalue. The entire dependence on the crystal momentum $\boldsymbol{k}$ is carried by the quantum Hall wave function $\Phi_{k}$, which exhibits one node at a $\boldsymbol{k}$-dependent position, while $\mathcal{G}$, which is independent of $\boldsymbol{k}$, has a number of nodes that increases as the magic angle index increases, indicating a similarity between higher magic angles and higher Landau levels. This structure is revealed in Fig. 1, which for the first three magic angles presents the norm of each component of $\phi_{\boldsymbol{k}}$ for the case where $\boldsymbol{k}$ is fixed at the moire Dirac point $\boldsymbol{K}$ and implies a charge variation that can be detected by scanning tunneling spectroscopy.

We show that Eq. (1) explains how the wave function $\phi_{k}$ can simultaneously have the Abelian translation symmetry of the usual Bloch wave function and give rise to the anomalous
Hall effect. Further, the quantum Hall antiquantum Hall structure implies that the wave function nodes have a chirality and we find that nodes of a given chirality are concentrated in particular regions of the unit cell, implying intracell circulating currents that grow in magnitude as the magic angle increases. The increased density of nodes at higher magic angles will also affect the project of electron-electron interactions onto the flat bands in a manner similar to that occurring at higher Landau levels in the quantum Hall problem.

The paper is organized as follows. Section II reviews the continuum model of twisted bilayer graphene and the chiral model defined from it, to establish the notation and approximations used here. Section III introduces our intravalley inversion symmetry and derives some of the properties that follow from it. Then in Sec. IV, we reexamine the derivation of the flat-band wave functions and derive their spinor structure. We then discuss the nodal structure of the flat-band 

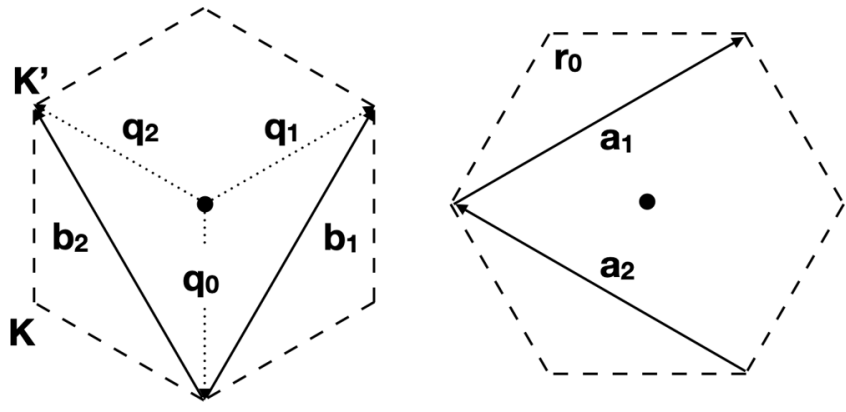

FIG. 2. (Left) Moire Brillouin zone. Right: real-space moire unit cell. Shown are the reciprocal lattice vectors $\boldsymbol{b}_{1,2}$, the moire Dirac points $\boldsymbol{K}, \boldsymbol{K}^{\prime}$, the real-space lattice vectors $\boldsymbol{a}_{1,2}$ and the wave vectors $\boldsymbol{q}_{0,1,2}$, and the $B A$ stacking point $\boldsymbol{r}_{0}$. The $A B$ and AA stacking points are located respectively at $-\boldsymbol{r}_{0}$ and the origin of the unit cell.

wave functions in Sec. V. In the last part of this work, Sec. VI, we discuss how our findings can impact experimental observables. Section VII is a summary and conclusions.

\section{MODEL HAMILTONIANS}

We start this section by reviewing the continuum model [1-3] and the chiral model [52] of twisted bilayer graphene to establish the notation.

When two parallel graphene sheets (top, bottom) are stacked with any one of an infinite set of relative commensurate angles $\theta$, a moire pattern forms, in which the combined system retains the basic hexagonal lattice structure of graphene, but with a much larger unit cell containing a number of carbon atoms $\sim \theta^{-2}$. The corresponding reciprocal space unit cell, which we refer to as the moire Brillouin zone, is illustrated in Fig. 2.

As shown in Fig. 2, $\boldsymbol{a}_{i=1,2}$ indicate the two dimensional basis vectors of the moire unit cell. The area of the moire unit cell is $2 \pi S=\left|\boldsymbol{a}_{1} \times \boldsymbol{a}_{2}\right|$. We denote the reciprocal lattice vectors as $\boldsymbol{b}^{i=1,2}$. Throughout this paper, we define the unit length by setting $\sqrt{S}=1$.

The fundamental single-particle Hamiltonian for twisted bilayer graphene consists of a standard single-layer graphene Hamiltonian for the top/bottom layer, $h_{G}\left(\boldsymbol{r}, \boldsymbol{r}^{\prime}\right)$, and an interlayer coupling $T\left(\boldsymbol{r}, \boldsymbol{r}^{\prime}\right)$ whose periodicity defines the moire superlattice. Schematically the Hamiltonian operator is

$$
H_{T B L G}\left(\boldsymbol{r}, \boldsymbol{r}^{\prime}\right)=\left(\begin{array}{cc}
h_{G}^{b}\left(\boldsymbol{r}, \boldsymbol{r}^{\prime}\right) & T\left(\boldsymbol{r}, \boldsymbol{r}^{\prime}\right) \\
T^{\dagger}\left(\boldsymbol{r}, \boldsymbol{r}^{\prime}\right) & h_{G}^{t}\left(\boldsymbol{r}, \boldsymbol{r}^{\prime}\right)
\end{array}\right) .
$$

where $t / b$ stands for the top/bottom graphene sheet.

It is generally agreed that, as proposed by Bistritzer and MacDonald [1], the low energy properties of twisted bilayer graphene can be adequately described by a model with three key features. The first is a continuum description of the physics in each graphene sheet, obtained by linearizing the graphene Hamiltonian $h_{G}$ near the Dirac points (we denote the linearized Dirac Hamiltonian as $h_{D}$ ). The second is that the interlayer hopping only couples states near one Dirac point in one layer with states near the same graphene Dirac point in the other layer. This means that the relevant Hamiltonian is the product of two copies, one for each valley.
A third simplification proposed by Bistritzer and MacDonald is that the interlayer hopping, in principle a function of $\boldsymbol{r}$ in one layer and $\boldsymbol{r}^{\prime}$ in the other becomes a function only of $\boldsymbol{r}$ with $\boldsymbol{r}^{\prime}=\boldsymbol{r}$. This is a coarse-graining approximation based on the notion that $T(\boldsymbol{r})$ has a range of the order of the carbon-carbon distance so if the wave functions vary slowly on this scale we can ignore the detailed local structure.

Following Bistritzer and MacDonald [1], the effective continuum Hamiltonian of a single valley is

$$
H_{\mathrm{BM}}=\int d^{2} \boldsymbol{r} \Psi_{\mathrm{BM}}^{\dagger}(\boldsymbol{r})\left(\begin{array}{cc}
h_{D}^{b}\left(\frac{\theta}{2}\right) & T(\boldsymbol{r}) \\
T^{\dagger}(\boldsymbol{r}) & h_{D}^{t}\left(-\frac{\theta}{2}\right)
\end{array}\right) \Psi_{\mathrm{BM}}(\boldsymbol{r}) .
$$

A related Hamiltonian can be found for the opposite valley by acting with time reversal symmetry. The wave function $\Psi_{\mathrm{BM}}(\boldsymbol{r})$ is a four-component spinor, with the lower two components the two sublattices of the top layer, and the upper two the two sublattices of the bottom layer:

$$
\Psi_{\mathrm{BM}}(\boldsymbol{r})=\left(\begin{array}{c}
\psi_{b}^{A}(\boldsymbol{r}) \\
\psi_{b}^{B}(\boldsymbol{r}) \\
\psi_{t}^{A}(\boldsymbol{r}) \\
\psi_{t}^{B}(\boldsymbol{r})
\end{array}\right) .
$$

We have suppressed the spin index because the global $S U(2)$ spin invariance implies that the single-particle Hamiltonian is spin-diagonal. The continuum approximation to the Dirac Hamiltonian of a layer $\lambda=t, b$ is

$$
h_{D}^{\lambda}\left(\frac{\theta}{2}\right)=v_{0}\left(-i \nabla-\boldsymbol{K}_{+}^{\lambda}\right) \cdot e^{-\frac{i \theta}{4} \sigma_{z}} \boldsymbol{\sigma} e^{\frac{i \theta}{4} \sigma_{z}},
$$

where $\boldsymbol{K}_{+}^{t / b}$ is the graphene Dirac point $\boldsymbol{K}_{+}$rotated by $\pm \theta / 2$. As shown in Fig. 2, we define the moire Dirac points as $\boldsymbol{K}=$ $\boldsymbol{K}_{+}^{b}-\boldsymbol{K}_{+}^{\Gamma}, \boldsymbol{K}^{\prime}=\boldsymbol{K}_{+}^{t}-\boldsymbol{K}_{+}^{\Gamma}$ where $\boldsymbol{K}_{+}^{\Gamma}$ is the moire Gamma point labeled in graphene's reciprocal lattice coordinates. The interlayer tunneling potential $T(\boldsymbol{r})$ is constrained by the symmetries of a single valley: $\mathcal{C}_{3}, \mathcal{M}_{y}$ and $\mathcal{C}_{2} \mathcal{T}$, as discussed in Sec. III A. In the Bistritzer-MacDonald model, the interlayer hopping is

$$
T(\boldsymbol{r})=\sum_{j=0}^{2} T_{j} e^{-i\left(\boldsymbol{q}_{0}-\boldsymbol{q}_{j}\right) \cdot \boldsymbol{r}}
$$

with $\phi=2 \pi / 3$, the $T_{j}$ is

$$
T_{j}=\omega_{0}-\omega_{1} \cos (j \phi) \sigma_{x}+\omega_{1} \sin (j \phi) \sigma_{y} .
$$

The chiral model [52] is obtained by setting $\omega_{0}=0$ in Eq. (7). The chiral model for a single valley is written in a different basis as $H_{\mathrm{BM}}$ in Eq. (3):

$$
H_{\mathrm{cBM}}=\int d^{2} \boldsymbol{r} \Psi_{c}^{\dagger}(\boldsymbol{r})\left(\begin{array}{cc}
0 & \mathcal{D}(\boldsymbol{r}) \\
\mathcal{D}^{\dagger}(\boldsymbol{r}) & 0
\end{array}\right) \Psi_{c}(\boldsymbol{r}),
$$

where $\Psi_{c}(\boldsymbol{r})$ is a four-component spinor whose upper two components $(\phi)$ correspond to the A sublattice of the bottom and top layers, and the lower two components $(\chi)$ the B sublattice of the bottom and top layers:

$$
\Psi_{c}(\boldsymbol{r})=\left(\begin{array}{c}
\phi_{b}(\boldsymbol{r}) \\
\phi_{t}(\boldsymbol{r}) \\
\chi_{b}(\boldsymbol{r}) \\
\chi_{t}(\boldsymbol{r})
\end{array}\right),
$$


where we have suppressed the Bloch momentum $\boldsymbol{k}$. The unitary transformation between the nonchiral basis Eq. (4) and the chiral basis Eq. (9) is

$$
\Psi_{c, \boldsymbol{k}}(\boldsymbol{r})=e^{-i\left(\boldsymbol{K}_{+}^{\Gamma}+\tau_{z} \boldsymbol{K}\right) \cdot \boldsymbol{r}} e^{i \frac{\theta}{4} \tau_{z} \sigma_{z}} \Psi_{\mathrm{BM}, \boldsymbol{K}_{+}^{\Gamma}+\boldsymbol{k}}(\boldsymbol{r}),
$$

where in Eq. (10), we have used $\sigma$ and $\boldsymbol{\tau}$ for Pauli matrices acting on the sublattice and layer degrees of freedom, respectively,

$$
\sigma: \text { sublattices; } \boldsymbol{\tau} \text { : layers. }
$$

In Eq. (10), we have also shifted the center of the Bloch momentum of the chiral basis to the moire Gamma point. The Bloch boundary condition of the chiral basis is

$$
\Psi_{c, \boldsymbol{k}}(\boldsymbol{r}+\boldsymbol{a})=e^{i\left(\boldsymbol{k}-\tau_{z} \boldsymbol{K}\right) \cdot \boldsymbol{a}} \Psi_{c, \boldsymbol{k}}(\boldsymbol{r}),
$$

where the details of Eq. (10) and Eq. (11) can be found in Appendix A.

The operators $\mathcal{D}^{\dagger}(\boldsymbol{r})$ and $\mathcal{D}(\boldsymbol{r})$ in Eq. (8) are

$$
\begin{aligned}
\mathcal{D}^{\dagger}(\boldsymbol{r}) & =\sqrt{2}\left(\begin{array}{cc}
-i \bar{\partial} & \alpha U_{\phi}(\boldsymbol{r}) \\
\alpha U_{\phi}(-\boldsymbol{r}) & -i \bar{\partial}
\end{array}\right), \\
\mathcal{D}(\boldsymbol{r}) & =\sqrt{2}\left(\begin{array}{cc}
-i \partial & \alpha U_{-\phi}(\boldsymbol{r}) \\
\alpha U_{-\phi}(-\boldsymbol{r}) & -i \partial
\end{array}\right),
\end{aligned}
$$

where $U_{\phi}(\boldsymbol{r})$ is

$$
U_{\phi}(\boldsymbol{r})=e^{-i \boldsymbol{q}_{0} \cdot \boldsymbol{r}}+e^{i \phi} e^{-i \boldsymbol{q}_{1} \cdot \boldsymbol{r}}+e^{-i \phi} e^{-i \boldsymbol{q}_{2} \cdot \boldsymbol{r}} .
$$

As usual, we have defined $z=(x+i y) / \sqrt{2}, \partial=\left(\partial_{x}-\right.$ $\left.i \partial_{y}\right) / \sqrt{2}$. The parameter $\alpha$ is determined by the twisted angle: $\alpha=\left(3 w_{1} a_{0}\right) /\left(8 \sqrt{2} \pi v_{0} \sin \frac{\theta}{2}\right)$ where $v_{0}$ is the graphene's Fermi velocity and $a_{0}$ is the graphene's lattice constant. The vectors $\boldsymbol{q}_{0,1,2}$ are specified in Fig. 2 .

The chiral Hamiltonian anticommutes with the chiral matrix $\sigma_{z}$. As a consequence, the single-particle spectrum is particle-hole symmetric. In the next section, we review symmetries of twisted bilayer graphene, and introduce the intravalley inversion symmetry.

\section{INTRAVALLEY INVERSION SYMMETRY}

In this section, we start by discussing the symmetries of twisted bilayer graphene with an emphasis on how $\mathcal{C}_{2} \mathcal{T}$ symmetry constrains the tunneling terms. In Sec. III B, we introduce the exact intravalley inversion symmetry of the chiral model, and derive some properties that follow from it.

\section{A. Symmetry constraint on tunneling terms}

The symmetries of twisted bilayer graphene play crucial roles in determining the single and many particle properties [30-47]. In this section, we review these symmetries, with an emphasis on how symmetries constrain the low energy continuum Hamiltonian.

The "crystal symmetries" of twisted bilayer graphene are generated by the moire translation symmetry, $\mathcal{C}_{6}$ rotational rotation, and mirror symmetry $\mathcal{M}_{y}$. Time reversal symmetry, $\mathcal{T}$, is also present. In addition, in the continuum model the charge conservation of each valley, i.e., U(1) valley symmetry, is assumed. The symmetries that keep each valley invariant $\left(\mathcal{C}_{2} \mathcal{T}, \mathcal{C}_{3}\right.$, and $\left.\mathcal{M}_{y}\right)$ constrain the single valley Hamiltonian in
Eqs. (3) and (8). Here, the important constraint for us is that $\mathcal{C}_{2} \mathcal{T}$ symmetry requires the tunneling term in Eq. (3) satisfy (proof in Appendix B):

$$
T(\boldsymbol{r})=\sigma_{x} T^{*}(-\boldsymbol{r}) \sigma_{x},
$$

where, as in the previous section, $\sigma_{x}$ acts on sublattice space. In the chiral basis, this means that the off-diagonal elements of $\mathcal{D}$ (and $\mathcal{D}^{\dagger}$ ) are related by $\boldsymbol{r} \leftrightarrow-\boldsymbol{r}$, as we shown in Eq. (12).

\section{B. Exact intravalley inversion symmetry of the chiral model}

Here we show that the chiral model enjoys an exact intravalley inversion symmetry as constrained by $\mathcal{C}_{2} \mathcal{T}$ symmetry and the linearized Dirac fermion. We then discuss properties that follow from it, including the symmetries of the spectrum and single-particle states. In the end, we show a numerically observed alternating pattern of magic angle inversion parities.

Lemma 1. The zero-mode operator satisfies

$$
\tau_{y} \mathcal{D}^{\dagger}(\boldsymbol{r}) \tau_{y}=-\mathcal{D}^{\dagger}(-\boldsymbol{r})
$$

The calculation follows from the $\mathcal{C}_{2} \mathcal{T}$ constraint in Eq. (14) and the definition of $\mathcal{D}^{\dagger}$ in Eq. (12). For Lemma 1 to hold, we need the off-diagonal elements of the $\mathcal{D}(\boldsymbol{r})$ operator to be related by $\boldsymbol{r} \leftrightarrow-\boldsymbol{r}$. In other words, in the chiral basis [Eq. (9)], the interlayer tunneling potential from top to bottom layer is identical to that from bottom to top layer with spatial inversion. As we discussed in Sec. III A, this is guaranteed by the $\mathcal{C}_{2} \mathcal{T}$ symmetry.

We now define the intravalley inversion symmetry.

Theorem 1. The chiral model of twisted bilayer graphene has an exact intravalley inversion symmetry, whose operator is

$$
\mathcal{I} \equiv \sigma_{z} \tau_{y}
$$

such that

$$
\mathcal{I} \mathcal{H}(\boldsymbol{r}) \mathcal{I}^{\dagger}=\mathcal{H}(-\boldsymbol{r}) .
$$

Again, $\sigma$ and $\tau$ are Pauli matrices acting on the sublattice and layer degrees of freedom, respectively.

Proof. It is straightforward to prove by using Lemma 1:

$$
\begin{aligned}
\mathcal{I H}(\boldsymbol{r}) \mathcal{I}^{\dagger} & =\left(\begin{array}{cc}
\tau_{y} & 0 \\
0 & -\tau_{y}
\end{array}\right)\left(\begin{array}{cc}
0 & \mathcal{D}(\boldsymbol{r}) \\
\mathcal{D}^{\dagger}(\boldsymbol{r}) & 0
\end{array}\right)\left(\begin{array}{cc}
\tau_{y} & 0 \\
0 & -\tau_{y}
\end{array}\right) \\
& =-\left(\begin{array}{cc}
0 & \tau_{y} \mathcal{D}(\boldsymbol{r}) \tau_{y} \\
\tau_{y} \mathcal{D}^{\dagger}(\boldsymbol{r}) \tau_{y} & 0
\end{array}\right)=\mathcal{H}(-\boldsymbol{r}) .
\end{aligned}
$$

We call Eq. (17) the intravalley inversion symmetry in order to distinguish it from the crystalline 2D inversion symmetry, $\mathcal{C}_{2}$. Since the $\mathcal{C}_{2}$ symmetry mixes valleys of twisted bilayer graphene, it is not a symmetry of the single-valley continuum models in Eqs. (3) and (8). In contrast, the intravalley inversion maps $\boldsymbol{k}$ to $-\boldsymbol{k}$ within the moire Brillouin zone and thus does not mix valleys. As shown in Eq. (17), the intravalley inversion symmetry is an exact symmetry for the single valley chiral model (8).

We emphasize that the intravalley inversion symmetry requires no extra assumptions beyond the chiral model in Eq. (8). The only requirement is the crystal symmetry $\mathcal{C}_{2} \mathcal{T}$ 
and the linearized Dirac fermion, which are already present in the chiral model in Eq. (8).

The $\tau_{y}$ operator of Eq. (15) has appeared in recent literature. In Ref. [54], it is referred to as the involution operator. It also appeared as Eq. (S15) in the supplementary material of Ref. [41]. In Ref. [35] and a very recent paper Ref. [43], by the same authors, a similar operator $i \tau_{y}$ plus $\boldsymbol{r} \leftrightarrow-\boldsymbol{r}$ is termed the unitary particle-hole operator. This is different than our intravalley inversion symmetry: our $\tau_{y}$ operates on the chiral basis in Eq. (9), while the "unitary particle-hole" acts on the nonchiral basis in Eq. (4). Since the unitary transformation between these two bases in Eq. (10) does not commute with $\tau_{y}$, these two symmetries are distinct. It is also important to emphasize that our intravalley inversion is an exact symmetry of the chiral model, while the unitary particle-hole symmetry is an approximate symmetry for both the continuum model in Eq. (3) and the chiral model in Eq. (8), according to Refs. [35] and [43].

Many interesting facts follow from the intravalley inversion symmetry, as we describe here and in the next section.

Corollary 1. At all twist angles, the single particle spectrum of the chiral model is not only particle-hole symmetric, but also inversion symmetric.

This follows directly from Theorem 1 . Denote the sublattice $\mathrm{A} / \mathrm{B}$ wave functions as

$$
\Psi_{k}=\left(\begin{array}{l}
\phi_{k} \\
\chi_{k}
\end{array}\right)
$$

where each of $\phi_{k}$ and $\chi_{k}$ is a two-component spinor representing the bottom and top layer's degrees of freedom. If we know $\Psi_{k}$ as an eigenstate of energy $E$ at Bloch momentum $\boldsymbol{k}$, then $\mathcal{I} \Psi_{k}(-\boldsymbol{r})$ is the eigenstate of the same energy but with an opposite Bloch momentum:

$$
\mathcal{H}(\boldsymbol{r}) \mathcal{I} \Psi_{k}(-\boldsymbol{r})=\mathcal{I} \mathcal{H}(-\boldsymbol{r}) \Psi_{k}(-\boldsymbol{r})=E \mathcal{I} \Psi_{k}(-\boldsymbol{r}) .
$$

We have thus proved the spectrum inversion symmetry by explicitly constructing eigenstates of the same energy and opposite Bloch momentum. This construction in fact also illustrates a spinor structure of the eigenstates.

Theorem 2. At all twist angles for any Bloch momentum $\boldsymbol{k}$, there exists a phase $\zeta_{k}$, such that,

$$
\begin{aligned}
\phi_{k}(\boldsymbol{r}) & =+e^{i \zeta_{k}} \tau_{y} \phi_{-\boldsymbol{k}}(-\boldsymbol{r}), \quad \chi_{k}(\boldsymbol{r})=-e^{i \zeta_{k}} \tau_{y} \chi_{-\boldsymbol{k}}(-\boldsymbol{r}), \\
\zeta_{k} & =-\zeta_{-\boldsymbol{k}} .
\end{aligned}
$$

Proof. Below Corollary 1, we explicitly constructed the eigenstate of opposite Bloch momentum. At nondegenerate $\boldsymbol{k}$, our constructed wave function must be proportional to the wave function at $-\boldsymbol{k}$ up to a $\mathrm{U}(1)$ phase,

$$
\Psi_{-k}(\boldsymbol{r})=e^{i \zeta_{k}} \sigma_{z} \tau_{y} \Psi_{k}(-\boldsymbol{r}),
$$

from which Eq. (19) follows immediately. The fact that the phase $\zeta_{k}$ is antisymmetric is seen by applying Eq. (19) twice. For degenerate zero modes, one can label them by the chiral eigenvalue and find the same conclusion.

Theorem 2 can be regarded as a gauge fixing condition. One can perform gauge transformations

$$
\phi_{k} \rightarrow e^{i \zeta_{k}^{\prime}} \phi_{k}
$$

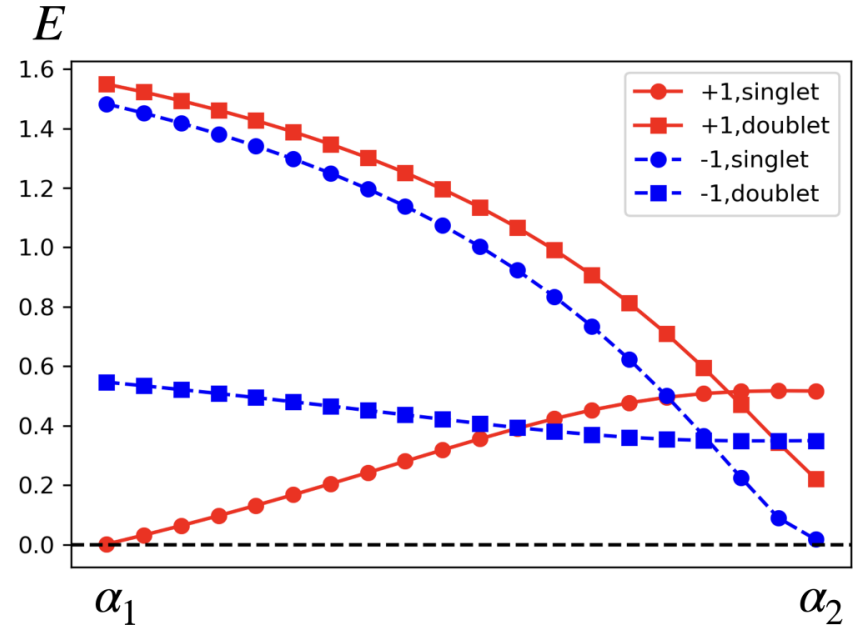

FIG. 3. Evolution at the moire Gamma point of low lying nonnegative energy states from the first magic angle $\alpha_{1}$ to the second magic angle $\alpha_{2}$. A singlet (doublet) state is represented as dots (squares). The inversion symmetric (antisymmetric) state is represented by red solid (blue dashed) lines. The black horizontal line indicates zero energy. Due to the particle-hole symmetry of the chiral model, the evolution of negative energy states is obtained by reflecting the figure.

to tune the $\zeta_{k}$ field:

$$
\zeta_{ \pm k} \rightarrow \zeta_{ \pm k} \mp\left(\zeta_{k}^{\prime}-\zeta_{-k}^{\prime}\right)
$$

The only obstruction of such tuning is at inversion symmetric points where $\boldsymbol{k}_{i n v}=-\boldsymbol{k}_{i n v}$ modulo reciprocal lattice vectors: there $\zeta_{\boldsymbol{k}}^{\prime}$ and $\zeta_{-\boldsymbol{k}}^{\prime}$ cancel, and $\zeta_{\boldsymbol{k}_{i v v}}$ is either 0 or $\pi$. In practice, the intravalley inversion eigenvalue can be read off from the transformation property of the moire Gamma point wave function (or from wave functions at other $\boldsymbol{k}_{\text {inv }}$ ):

$$
\phi_{k=0}(\boldsymbol{r})=\eta \tau_{y} \phi_{k=0}(-\boldsymbol{r}), \quad \eta= \pm 1 .
$$

We numerically observed (for the first three magic angles) that there is a coincidence between the zero mode's intravalley inversion eigenvalue and the parity of magic angle: we found $\eta=+1$ for the first, third magic angles, while for the second magic angle $\eta=-1$. In Fig. 3, we monitored the evolution of the low lying eigenstates at the Gamma point from the first to the second magic angle (we plot non-negative energies only since the full spectrum has particle-hole symmetry). Eigenstates at the Gamma point are either singlet or doublet, as they are one and two-dimensional irreducible representation of the symmetry group (generated by $\mathcal{C}_{3}$ and $\mathcal{M}_{y}$ ) [33]. An inversion eigenvalue transition is clearly visible in Fig. 3.

We hypothesize that the alternating parity of magic angle zero-mode wave functions is a generic feature and will hold for all magic angles: that is, the inversion eigenvalue of $n_{t h}$ magic angle flat-band wave function is $-(-1)^{n}$.

\section{ZERO-MODE WAVE FUNCTIONS AND THE SPINOR STRUCTURE}

In this section, we reexamine the zero-mode solution of Ref. [52]), derive the spinor structure of the zero-mode wave 
function as shown in Eq. (1), and show its intricate relation to quantum Hall physics.

\section{A. Chiral model and the zero modes}

Following Ref. [52]), we show that the chiral Hamiltonian in Eq. (8) has two zero modes. The eigenvectors of these two zero modes must satisfy:

$$
0=\mathcal{D}(\boldsymbol{r})\left(\begin{array}{c}
\chi_{b, k}(\boldsymbol{r}) \\
\chi_{t, k}(\boldsymbol{r})
\end{array}\right) ; \quad 0=\mathcal{D}^{\dagger}(\boldsymbol{r})\left(\begin{array}{c}
\phi_{b, \boldsymbol{k}}(\boldsymbol{r}) \\
\phi_{t, \boldsymbol{k}}(\boldsymbol{r})
\end{array}\right) .
$$

In Ref. [52]), the authors found and proved that, for a discrete series of values of $\alpha$ (corresponding to magic angles), the chiral model admits exact flat bands. At the crux of their analysis is the fact that at magic angles, both components of the moire Dirac $\boldsymbol{K}$ point wave function $\phi_{\boldsymbol{K}}=\left(\phi_{b, \boldsymbol{K}}, \phi_{t, \boldsymbol{K}}\right)^{T}$ vanish at a common point:

$$
\boldsymbol{r}_{0}=\frac{1}{3}\left(\boldsymbol{a}_{1}+2 \boldsymbol{a}_{2}\right)
$$

the $B A$ stacking point, which permits an explicit construction of the zero-mode wave functions. Here we review several key steps (Theorem 3-5) of Ref. [52] in deriving the zero-mode wave functions. We refer the readers to Ref. [52] for more details.

A crucial step in deriving the zero-mode solutions in Ref. [52] is Theorem 3, which follows from the translation and $\mathcal{C}_{3}$ rotation symmetry:

Theorem 3. For all twisted angles, $\phi_{\boldsymbol{K}, t}\left( \pm \boldsymbol{r}_{0}\right)=0$ and $\chi_{\boldsymbol{K}, t}\left( \pm \boldsymbol{r}_{0}\right)=0$.

Theorem 4. The Fermi velocity defined by

$$
v_{F}(\alpha) \equiv \sum_{l=t / b} \phi_{l, \boldsymbol{K}}(\boldsymbol{r}) \phi_{l, \boldsymbol{K}}(-\boldsymbol{r})
$$

is independent of $\boldsymbol{r}$.

Proof. It is straightforward to find $v_{F}(\alpha)$ is holomorphic, i.e., $\bar{\partial} v_{F}(\alpha)=0$, by using the zero-mode equations that $\phi_{l, \boldsymbol{K}}$ satisfy. Then, $v_{F}(\alpha)$ must be a constant since it is also cellperiodic.

At magic angles (where the low lying two bands become dispersionless), the Fermi velocity goes to zero. Since the top component of $\phi_{\boldsymbol{K}}$ vanishes for all twist angles at $\pm \boldsymbol{r}_{0}$, it follows from the vanishing Fermi velocity that the bottom component must at least have one common zero with the top component, at either $+\boldsymbol{r}_{0}$ or $-\boldsymbol{r}_{0}$. In fact, the exact flat-band condition coincides with the condition that two components of the wave function have a common zero, as pointed out in Ref. [52], where the authors proved it by explicitly constructing the zero-mode wave functions.

Theorem 5. The magic angle zero-mode wave functions take the following form [52,55] (up to a normalization factor):

$$
\phi_{\boldsymbol{k}}(\boldsymbol{r})=\phi_{\boldsymbol{K}}(\boldsymbol{r}) F_{\boldsymbol{k}}(z), \quad F_{\boldsymbol{k}}(z)=e^{z_{k}^{*}\left(z-\frac{1}{2} z_{k}\right)} \frac{\sigma\left(z-z_{k}\right)}{\sigma\left(z-z_{0}\right)},
$$

where $z_{0}$ and $z_{k}$ are the complex coordinates of $\boldsymbol{r}_{0}$, the $B A$ stacking point defined in Eq. (24), and

$$
\boldsymbol{r}_{\boldsymbol{k}}^{a}=\boldsymbol{r}_{0}^{a}+\epsilon^{a b}(\boldsymbol{k}-\boldsymbol{K})_{b}
$$

The complex coordinate for a vector $\boldsymbol{r}$ is defined as usual

$$
r \rightarrow z \equiv \frac{r_{x}+i r_{y}}{\sqrt{2}} .
$$

Note here we have written the zero-mode wave function in terms of the "modified Weierstrass sigma" function $\sigma(z)$, which is slightly different from Ref. [52]), where the authors used Jacobi theta functions. It has been shown [56-59] that both the sigma function and theta function can be used to define the quantum Hall states, and the advantage of the former is modular invariance. The Weierstrass sigma function satisfies a similar quasiperiodic boundary condition as the Jacobi theta function:

$$
\sigma\left(z+a_{i}\right)=-e^{a_{i}^{*}\left(z+\frac{1}{2} a_{i}\right)} \sigma(z)
$$

where $a_{i=1,2}$ are the complex coordinates of the primitive lattice vectors $\boldsymbol{a}_{1,2}$ shown in Fig. 2 . The quantum Hall wave function and the modified Weierstrass sigma function $\sigma(z)$ are reviewed in detail in Appendix C. Note that the factor $\exp \left(-\frac{1}{2}\left|z_{k}\right|^{2}\right)$ in Eq. (26) is needed to ensure that the normalization is periodic in $\boldsymbol{k}$.

The presence of the quasiperiodic elliptic function in the zero-mode solution is reminiscent of the lowest Landau level physics on torus $[53,60]$. We find it conceptually and practically advantageous to rewrite Eq. (26) in the following form, as a product of a quantum Hall wave function and a quasiperiodic spinor wave function:

$$
\phi_{\boldsymbol{k}}(\boldsymbol{r})=\left(\begin{array}{l}
\mathcal{G}_{1}(\boldsymbol{r}) \\
\mathcal{G}_{2}(\boldsymbol{r})
\end{array}\right) \times \Phi_{k}(\boldsymbol{r}),
$$

where $\mathcal{G}_{1 / 2}(\boldsymbol{r}) \equiv \phi_{\boldsymbol{K}, b / t}(\boldsymbol{r}) /\left(\sigma\left(z-z_{0}\right) e^{-\frac{1}{2}|z|^{2}}\right)$ and the quantum Hall wave function $\Phi_{k}$ is

$$
\Phi_{\boldsymbol{k}}(\boldsymbol{r})=e^{z_{k}^{*} z} \sigma\left(z-z_{k}\right) e^{-\frac{1}{2}\left|z_{k}\right|^{2}} e^{-\frac{1}{2}|z|^{2}},
$$

whose boundary condition can be found in Eq. (C14) in Appendix C. Reformulating the zero-mode wave function in this way makes the subsequent discussions in Sec. $\mathrm{V}$ more clear.

\section{B. Spinor structure of zero-mode wave functions}

The intravalley inversion implies that the two components of the (magic angle) zero-mode wave functions are not independent.

Theorem 6. The zero-mode wave function can be written as Eq. (1), which we copy below,

$$
\phi_{k}(\boldsymbol{r})=\left(\begin{array}{c}
i \mathcal{G}(\boldsymbol{r}) \\
\eta \mathcal{G}(-\boldsymbol{r})
\end{array}\right) \times \Phi_{k}(\boldsymbol{r}),
$$

where $\eta= \pm 1$ is the intravalley inversion eigenvalue from Eq. (22) and $\Phi_{\boldsymbol{k}}(\boldsymbol{r})$ is the quantum Hall wave function (31).

Proof. We start with the ansatz

$$
\phi_{k}(\boldsymbol{r})=\left(\begin{array}{l}
\mathcal{G}_{1}(\boldsymbol{r}) \\
\mathcal{G}_{2}(\boldsymbol{r})
\end{array}\right) \Phi_{k}(\boldsymbol{r}) .
$$

Applying Theorem 2 yields

$$
\phi_{k}(\boldsymbol{r})=e^{i \zeta_{k}}\left(\begin{array}{c}
i \mathcal{G}_{2}(-\boldsymbol{r}) \\
-i \mathcal{G}_{1}(-\boldsymbol{r})
\end{array}\right) \Phi_{k}(\boldsymbol{r})
$$

where we have used the inversion property of the quantum Hall wave function $\Phi_{-k}(-\boldsymbol{r})=-\Phi_{k}(\boldsymbol{r})$ (derived in Appendix C 3). Equating Eqs. (32) and (33) yields

$$
\phi_{k}(\boldsymbol{r})=\left(\begin{array}{c}
i \mathcal{G}(\boldsymbol{r}) \\
e^{i \zeta_{k}} \mathcal{G}(-\boldsymbol{r})
\end{array}\right) \Phi_{k}(\boldsymbol{r}), \quad e^{i \zeta_{k}}= \pm 1,
$$

where we defined $\mathcal{G}(\boldsymbol{r}) \equiv-i \mathcal{G}_{1}(\boldsymbol{r})$. 
The boundary condition of $\mathcal{G}(\boldsymbol{r})$ is derived in Eq. (C17) of Appendix C.

To conclude, following the intravalley inversion symmetry, we have derived the spinor structure of the zero-mode wave function as shown in Eq. (1) and have demonstrated explicitly its connection to the lowest Landau level wave functions. The $\eta$ in Eq. (1) is the intravalley inversion eigenvalue, which can be read off from Eq. (22).

\section{NODAL STRUCTURE}

In the previous sections, we described an intravalley inversion symmetry of the chiral model, which led to the discovery of the spinor structure of the zero-mode wave functions. There we factorized the wave function into a quantum Hall wave function and a pre-factor $\mathcal{G}(\boldsymbol{r})$.

However, so far the physical interpretation of the function $\mathcal{G}(\boldsymbol{r})$ remains mysterious, as does the structure of zeros in Fig. 1. One hint is that the zero modes must be Bloch functions that transform under the usual translation group, while quantum Hall states transform under the magnetic translation group. Hence $\mathcal{G}(\boldsymbol{r})$ must also be quasiperiodic to "cancel" the magnetic translation effects of the quantum Hall wave function.

In this section, we resolve this puzzle by demonstrating mathematically and numerically that $\mathcal{G}(\boldsymbol{r})$ can be regarded as an antiquantum Hall wave function at a certain Landau level, i.e., a quantum Hall state in a magnetic field oppositely directed to that of $\Phi_{k}$, with the order of the magic angle serving the role of the Landau level index. In this way, the zero-mode wave function is a product of a quantum Hall and an antiquantum Hall state, whose net magnetic fluxes passing through the moire unit cell cancel, allowing the whole wave function to be a usual Bloch function. We then discuss the zeros in more detail. In the next section, we discuss its experimental implications.

\section{A. Analytical expansion of $\mathcal{G}(r)$}

To demonstrate the antiquantum Hall nature of $\mathcal{G}(\boldsymbol{r})$, we will start by showing that the leading order expansion near $\boldsymbol{r}_{0}$ is antiholomorphic:

$$
\mathcal{G}\left(\boldsymbol{r}_{0}+\boldsymbol{r}\right) \sim \bar{z} .
$$

Hence we can peel off an antiquantum Hall wave function from the zero-mode wave function and rewrite its components as Eqs. (37) and (39).

Since $\mathcal{G}(\boldsymbol{r})$ is independent of Bloch momentum, without loss of generality we can consider the moire $\boldsymbol{K}$ point sublattice A wave function $\phi_{K}=\left(\phi_{K}^{b}, \phi_{K}^{t}\right)^{T}$ to analyze. Its zero-mode equation $\mathcal{D}^{\dagger}(\boldsymbol{r}) \phi(\boldsymbol{r})=0$ implies a relation between the top and bottom components $\phi_{K}^{t}=i \bar{\partial} \phi_{K}^{b} /\left(\alpha U_{\phi}\right)$. Theorem 3 tells us that $\phi_{K}^{t}$ must have zeros [52] at $\pm \boldsymbol{r}_{0}$. From the form of the zero-mode wave function Eq. (1), we know that the $+\boldsymbol{r}_{0}$ and $-\boldsymbol{r}_{0}$ zeros of $\phi_{\boldsymbol{K}}^{t}$ come, respectively, from the quantum Hall part $\Phi_{k}$ and $\mathcal{G}(-\boldsymbol{r})$. Therefore, near $\boldsymbol{r}_{0}, \phi_{\boldsymbol{K}}^{t}$ must vanish holomorphically:

$$
i \frac{\bar{\partial} \phi_{\boldsymbol{K}}^{b}\left(\boldsymbol{r}_{0}+\boldsymbol{r}\right)}{\alpha U_{\phi}\left(\boldsymbol{r}_{0}+\boldsymbol{r}\right)} \sim z
$$

Then, by using $U_{\phi}\left(\boldsymbol{r}_{0}\right)=3$ and the $\mathcal{C}_{3}$ symmetry, one can see that $\phi_{\boldsymbol{K}}^{b}$ must have a second order zero at $\boldsymbol{r}_{0}$, vanishing as: $\phi_{\boldsymbol{K}}^{b}\left(\boldsymbol{r}_{0}+\boldsymbol{r}\right) \sim z \bar{z}$. Again according to Eq. (1), $z$ and $\bar{z}$ of the bottom component $\phi_{K}^{b}$ come, respectively, from the quantum Hall wave function and $\mathcal{G}(\boldsymbol{r})$. We hence justified Eq. (35).

\section{B. Zero-mode wave function revisited}

The vanishing behavior of $\mathcal{G}(\boldsymbol{r})$ near $\boldsymbol{r}_{0}$ shows it is possible to factorize out an antiquantum Hall wave function [a quantum Hall state in a magnetic field oppositely directed to that of $\Phi_{k}$, which we denote as $\left.\bar{\Phi}_{k} \equiv\left(\Phi_{k}\right)^{*}\right]$ from it without encountering singularities. The Bloch momentum $\boldsymbol{k}$ of $\bar{\Phi}_{\boldsymbol{k}}$ is determined by the Bloch translation symmetry of the whole wave function. After some algebra, we end up with the final expression:

$$
\phi_{k}^{b}(\boldsymbol{r})=i \rho(\boldsymbol{r}) \times \bar{\Phi}_{\boldsymbol{K}}(\boldsymbol{r}) \Phi_{\boldsymbol{k}}(\boldsymbol{r}),
$$

where we introduced a function $\rho(\boldsymbol{r})$ which must be cellperiodic due to the cancellation of the nonperiodic parts from $\Phi_{k}$ and $\bar{\Phi}_{k}$ :

$$
\rho(\boldsymbol{r}) \equiv \mathcal{G}(\boldsymbol{r}) / \bar{\Phi}_{\boldsymbol{K}}(\boldsymbol{r}) .
$$

The top layer wave function is obtained easily by the intravalley inversion symmetry:

$$
\phi_{k}^{t}(\boldsymbol{r})=-\eta \rho(-\boldsymbol{r}) \times \bar{\Phi}_{\boldsymbol{K}^{\prime}}(\boldsymbol{r}) \Phi_{\boldsymbol{k}}(\boldsymbol{r}) .
$$

The $\Phi_{k}$ and $\bar{\Phi}_{K}$ of Eq. (37) carry opposite magnetic fields that cancel with each other, leaving $\phi_{k}^{b / t}$ as a Bloch state. Since the crystal momentum $(\boldsymbol{k})$ dependence, and hence response to an external electric field, is only from the $\Phi_{k}$ piece, the wave function $\phi_{k}$ should have the same topological character as the lowest Landau level wave function, according to Laughlin's gauge invariance argument [61].

To see how this argument applies to our case more explicitly, imagine we apply a time-independent and spatially uniform in-plane external electric field $\boldsymbol{E}$ across the twisted bilayer graphene sample. The Bloch momentum of the electron couples to $\boldsymbol{E}$ through minimal couping, and consequently changes linearly with time: $\delta \boldsymbol{k} \sim \boldsymbol{E} t$. According to Eq. (27), we know that the zero of $\Phi_{\boldsymbol{k}}$ is locked to $\boldsymbol{k}$, and moves in the direction perpendicular to $\boldsymbol{E}$. Since zero corresponds to a charge minimum, we conclude that a unit of charge is adiabatically pumped in a direction perpendicular to $\boldsymbol{E}$ during a unit of time. This demonstrates that the zero-mode wave function (1), as a product of a quantum Hall wave function and an antiquantum Hall wave function, is indeed a Bloch function which carries Chern number $\mathcal{C}=1$. So far, we discussed the sublattice-A polarized flat-band wave function $\phi_{k}(\boldsymbol{r})$. The other degenerate flat-band $\chi_{k}(\boldsymbol{r})$ is sublattice-B polarized and has Chern number $\mathcal{C}=-1$ since these two flat bands are related by the $\mathcal{C}_{2} \mathcal{T}$ symmetry before considering the hexagonal boron nitride substrate. With the hexagonal boron nitride substrate breaking the $\mathcal{C}_{2}$ symmetry, the two flat bands split in energy, and we expect a chiral gapless edge state connecting them. The time-reversal partner of the chiral edge would occur from the other valley with an opposite chirality. Although these two flat bands are sublattice polarized, the chiral edge mode is sublattice unpolarized since it connects two bulk bands of opposite sublattice polarization. 


\section{Zero structure}

We numerically observed that there are multiple zeros occurring at each order of magic angles, as shown in Fig. 1. We now demonstrate they are indeed zeros rather than numerical artifacts.

We noticed that all extra zeros occurring at higher magic angles are located at the reflection symmetric lines. The mirror symmetry $\mathcal{M}_{y}$ constrains that $\rho(x, y)$ and $\rho^{*}(x,-y)$ to be the same zero-mode solutions. By using the global U(1) phase degree of freedom of the wave function, $\rho(\boldsymbol{r})$ can be chosen to be a purely real function on the reflection symmetric line $y=0$, the red dotted line of Fig. 5. Here we parametrize this line by $\boldsymbol{r}=\lambda\left(\boldsymbol{a}_{1}-\boldsymbol{a}_{2}\right)=(x, 0)$ with $\lambda \in[-0.5,0.5)$, and plot $\rho(\lambda)$ along it in Fig. 4. Since $\rho(\lambda)$ is cell-periodic, it must cross zero along the reflection symmetric line an even number of times.

The zeros of the zero-mode wave functions are classified into two types by their "movability." One of them is a "movable zero" [62-64] from the quantum Hall wave function $\Phi_{k}$, whose location moves linearly with the Bloch wave vector:

$$
\boldsymbol{r}_{\boldsymbol{k}}^{a}=\boldsymbol{r}_{0}^{a}+\epsilon^{a b}(\boldsymbol{k}-\boldsymbol{K})_{b} .
$$

This zero carries the external Hall response of the Chern band. There are other "frozen zeros" whose locations are fixed and independent of the Bloch momentum $\boldsymbol{k}$. In particular, among these frozen zeros, one of them is from the antiquantum Hall state. In Fig. 5, we illustrate the zero-structure, where the black and blue dot represent the movable quantum Hall zero and the frozen antiquantum Hall zeros. The yellow and red dots are frozen zeros from the function $\rho(\boldsymbol{r})$.

Besides their "movability," zeros are also classified by their "chirality": the wave function receives a $2 \pi n$ phase when the coordinate $\boldsymbol{r}$ encircles the zero once anticlockwise. We numerically noticed that the black and red dots are $n=1$ zeros, while yellow and blue are $n=-1$ zeros. Interestingly, as shown in Fig. 1 the center of the unit cells are concentrated with more and more $n=-1$ zeros at higher magic angles. We discuss the implication for circulating currents in the next section.

We have shown that the zero-mode wave function shares some similarities with the simple harmonic oscillator system whose eigenstates also have alternating parity and have an increasing number of zeros. In Appendix D, we provide an analytical argument why these features might persist for all higher magic angles by an analogy to the harmonic oscillator [65].

\section{EXPERIMENTAL OBSERVATION AND IMPLICATIONS}

\section{A. Charge density and scanning tunneling probes}

One direct consequence of the zeros is a charge density deficiency that can be seen in scanning tunneling spectroscopy experiments $[36,66,67]$.

We expect a spectroscopy experiment will probe only the top (or bottom) layer, which corresponds to the components $\phi_{t}$ (top layer sublattice $\mathrm{A}$ wave function) and $\chi_{t}$ (top layer sublattice B wave function). If the spectroscopy measurement has spatial resolution on the level of the atomic spacing, then the sublattice wave functions can be probed separately. 1st magic angle

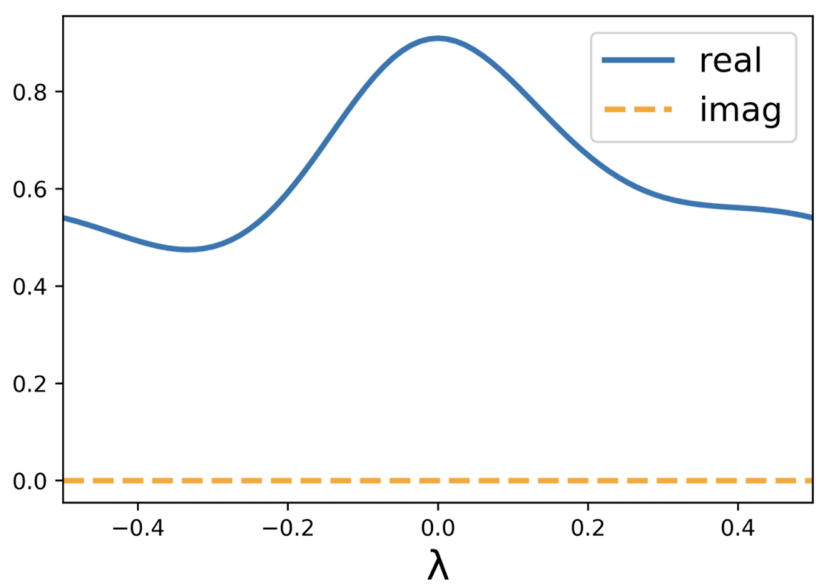

2nd magic angle

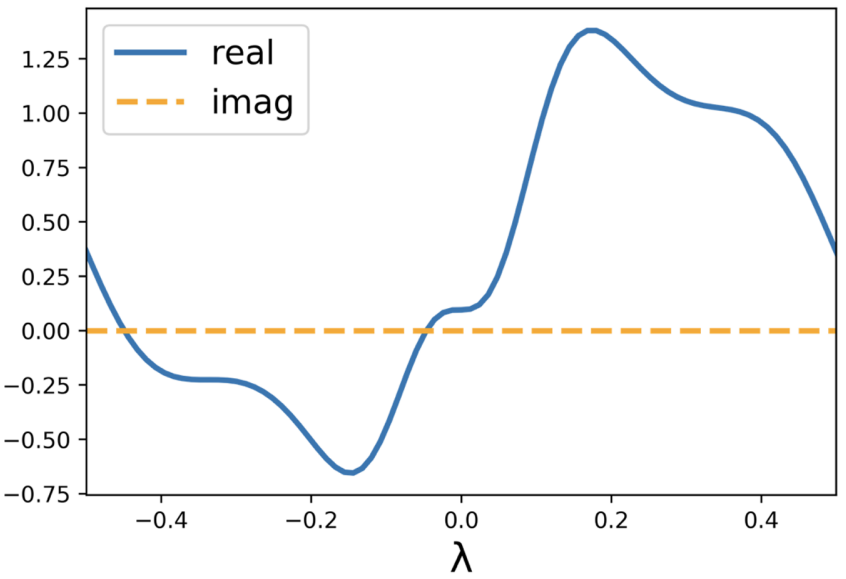

3rd magic angle

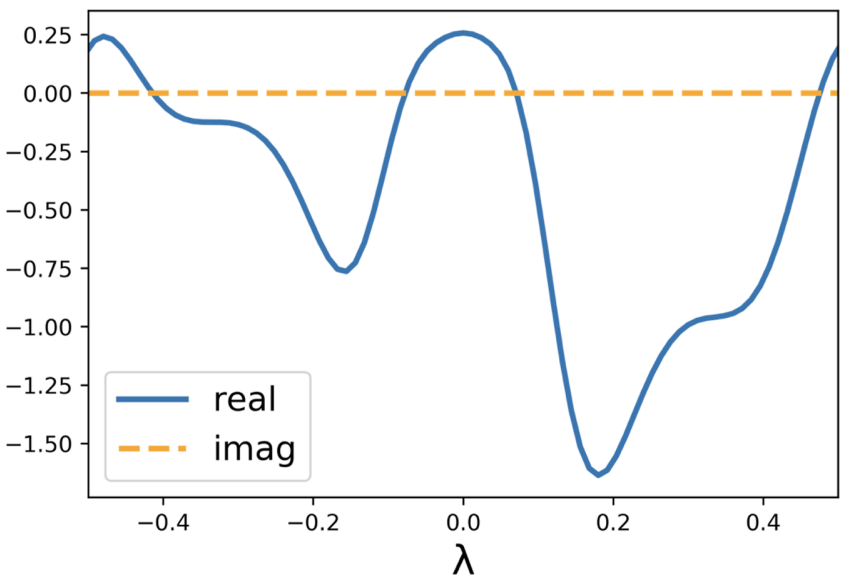

FIG. 4. Plot of $\rho(\boldsymbol{r})$ defined in Eq. (37) along the reflection symmetric line parameterized by $(x, 0)=\lambda\left(\boldsymbol{a}_{1}-\boldsymbol{a}_{2}\right)$ with $\lambda \in$ $[-0.5,0.5)$. The blue solid and orange dashed lines indicate the real and imaginary part of $\rho$ respectively. It can be seen from these plots that $\rho(\lambda)$ is real, and crosses zero an even number of times.

In this case, fixed zeros in the wave function components $\phi_{t}$ or $\chi_{t}$ correspond to the vanishing of charge density in real space, which will be strongly visible in the spectroscopy experiment. If the ground state is sublattice polarized, which 

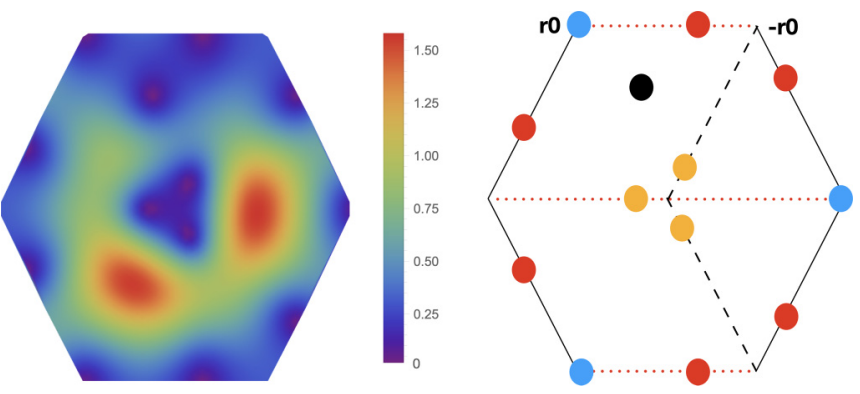

FIG. 5. Illustration of zeros. (Left) Real-space plot of the bottom component of the zero-mode wave function $\phi_{k}^{b}(\boldsymbol{r})$ at the second magic angle. Right: sketch of the zeros of the same wave function. Without loss of generality, we here choose $\boldsymbol{k}$ to be a generic point, $\boldsymbol{K}+0.3 \boldsymbol{b}_{1}-0.05 \boldsymbol{b}_{2}$. Since location of the black dot (the zero of the quantum Hall wave function $\Phi_{k}$ ) is locked with its Bloch momentum according to Eq. (40), it called a movable zero. The blue dot is the frozen zero from the antiquantum Hall wave function. The yellow and the red dots are the frozen zeros from the function $\rho(\boldsymbol{r})$. We found the black and red dots are $n=1$ zeros, while the yellow and blue dots are $n=-1$ zeros, where $n$ is the $2 \pi n$ phase that the wave function receives when the coordinate $\boldsymbol{r}$ encircles the zero once anticlockwise. The red dotted line is one of the three $\mathcal{C}_{3}$ symmetry related reflection symmetric lines.

maybe the case on a hexagonal boron nitride substrate, then such spatial resolution is not required to observe the zeros in a spectroscopy experiment. Notice that since the opposite valley wave function on the same sublattice is related by $\mathcal{T}$, which acts trivially in real space, we expect the two opposite valley wave functions on the same sublattice have the same location of fixed zeros. Therefore, probing the zeros with spectroscopy does not require valley polarization.

The accumulation of zeros at the unit cell center and the unit cell boundary as magic angle order increases, as shown in Fig. 1, should also be visible by spectroscopy with even less atomic resolution. This accumulation will become more prominent at higher magic angles.

Away from the chiral model, the zeros become nonzero minima in the charge density, which we have observed numerically. These will give a less sharp signature in scanning tunneling spectroscopy experiments, but will likely still be observable over some parameter regime.

\section{B. Higher Landau level physics at higher magic angles}

As we have seen from Sec. V, the $\mathcal{G}(\boldsymbol{r})$ piece of the flatband wave function in Eq. (1) has an increasing number of zeros and has an analytical expansion similar to an antiquantum Hall wave function. Consequently, we interpreted the zero-mode wave function as a product of a higher Landau level antiquantum Hall state and a lowest Landau level quantum Hall state [Eq. (37)], where the Landau level index of the former is determined by the order of the magic angle. We also discussed in Sec. VB that the topological properties of the flat bands are determined by the lowest Landau level quantum Hall piece $\Phi_{\boldsymbol{k}}$ since $\mathcal{G}(\boldsymbol{r})$ does not have Bloch momentum dependence.

Nevertheless, we expect the effective interactions projected into the flat bands are modified strongly by both $\mathcal{G}(\boldsymbol{r})$ and
$\Phi_{k}$. In particular, the nodal structure of $\mathcal{G}(\boldsymbol{r})$ directly impacts the charge density, which determines the projected Coulomb interaction. In the quantum Hall problem, the nodal structure of the higher Landau levels results in arrangement of charge that ultimately stabilizes various states [68-71] such as charge density waves, bubble phases, and other many-body topological phases (for instance, the non-Abelian Moore-Read phase [72]) that are absent in the lowest Landau level. By analogy, we might expect a different set of interacting phases to be stabilized at higher magic angles than at the first magic angle. Our formulation provides a theoretical and computational pathway towards analyzing interacting physics at different magic angles.

\section{Local current and magnetization at higher magic angles}

From the charge density of the zero-mode wave function as plotted in Fig. 1, we observe that for the first magic angle, the charge density maximum occurs at the unit cell center, i.e., the AA stacking point. At higher magic angles, we see an increasing number of zeros appearing at this region. Interestingly, all these zeros are of the same chirality for both layers, while zeros of the opposite chirality are pushed to the boundary of the unit cell. This indicates a stronger phase winding effect and hence circulating currents near the AA stacking region at higher magic angles, which could be experimentally observable nearby the chiral limit.

To see the circulation currents, we first define the following intrasublattice intralayer "current operator" $\boldsymbol{J}_{s S}^{l}$ for sublattice $s$ and layer $l$. The operator $\boldsymbol{J}_{A A}^{l}$ is defined as

$$
\boldsymbol{J}_{A A}^{l}(\boldsymbol{r}) \equiv i\left(t^{\prime}\right)\left[\left(\nabla \phi_{l}\right)^{*} \phi_{l}-\phi_{l}^{*}\left(\nabla \phi_{l}\right)\right](\boldsymbol{r}),
$$

operator $\boldsymbol{J}_{B B}^{l}(\boldsymbol{r})$ is defined in a similar manner but with $\phi$ replaced by $\chi$. Here $t^{\prime}$ is the microscopic parameter representing the next-nearest-neighbor hopping strength. We call the above a "current operator" in quotes because $\boldsymbol{J}_{s s}^{l}$ is not the current operator of the chiral model, which by definition should be proportional to $\partial_{k} H_{k}$, and hence couples distinct sublattices and vanishes within one sublattice. Since the exact flat-band wave functions are fully sublattice polarized (corresponding physically to hexagonal boron nitride splitting the sublattice degeneracy), the current operator $\partial_{k} H_{\boldsymbol{K}}$ vanishes within one sublattice polarized state. Nevertheless, we argue that our current operator $\boldsymbol{J}_{s s}^{l}$ has a microscopic origin, and hence should be a physical current operator. The $\boldsymbol{J}_{s s}^{l}$ can be regarded as a continuum version of lattice current $i\left(a_{s, i}^{\dagger} a_{s, j}-a_{s, j}^{\dagger} a_{s, i}\right)$ induced from the next-nearest-neighbor hopping process in graphene, where the $a_{s, i}$ are graphene's electron annihilation operators and $i, j$ labels graphene's nextnearest-neighbor sites.

In Fig. 6, we plot the real-space distribution of $\boldsymbol{J}_{A A}^{b}(\boldsymbol{r})$, calculated from the bottom layer sublattice-A wave function $\phi_{b}$ at three different Bloch momenta. According to Ref. [73]), the orbital magnetization is dominated by the Gamma point $\boldsymbol{K}_{0}$ and Dirac point $\boldsymbol{K}^{\prime}$ in a single valley model, since the bands hybridize most strongly with other bands at these points. Given the strong circulating current present at the second magic angle, it is reasonable to speculate a stronger orbital magnetization [74-80] at higher magic angles than the magnetization at the first magic angle $[73,81,82]$ for cases close 
First magic angle
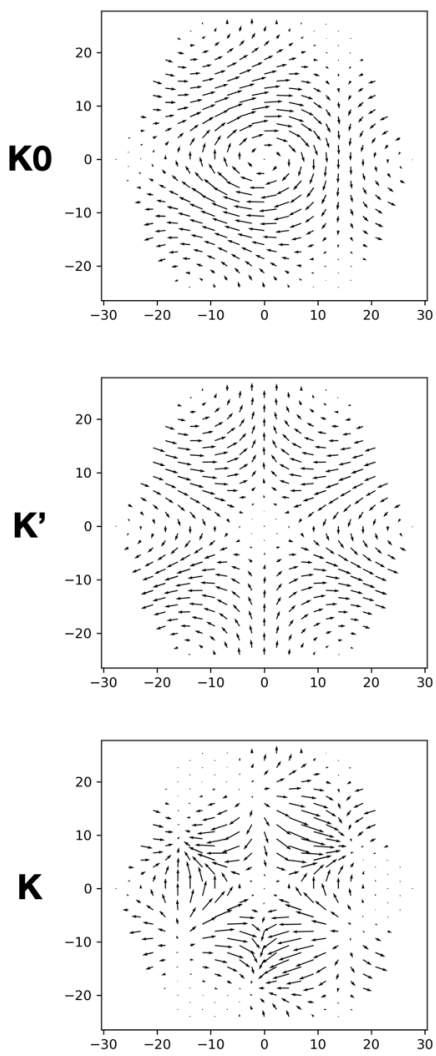

Second magic angle
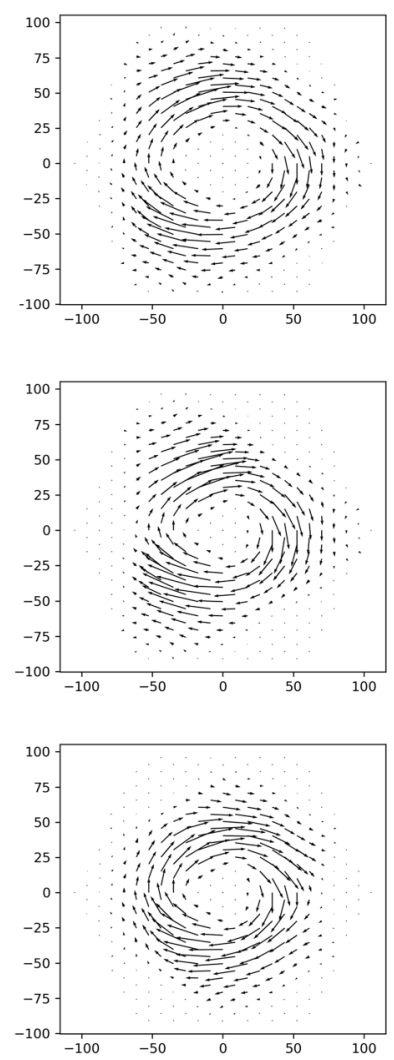

FIG. 6. Expectation value of the bottom layer sublattice A current operator $\boldsymbol{J}_{A A}^{b}$ at the moire Gamma point $\boldsymbol{K}_{0}$ and moire Dirac points $\boldsymbol{K}^{\prime}, \boldsymbol{K}$ at the first two magic angles. The chirality of the current operator would be opposite on the B sublattice, and the chirality is the same for both layers within one sublattice.

to the chiral limit. Note that the circulation currents are odd under time reversal or a sublattice transformation, hence a strong experimental signal requires valley and sublattice polarization. We leave a detailed exploration of higher magic angle orbital magnetization with more realistic parameters as future work.

\section{CONCLUSION}

In this work, we studied the chiral model of twisted bilayer graphene introduced in Ref. [52]). We pointed out the intrinsic intravalley inversion symmetry of the chiral model, protected by the $\mathcal{C}_{2} \mathcal{T}$ crystal symmetry and the linearized Dirac fermion. As a consequence, the energy spectrum is inversion symmetric at all twist angles. Furthermore, zero modes occurring at different magic angles are distinguished by their intravalley inversion eigenvalue. We numerically found a correspondence of the zero-mode inversion parity and the order of magic angles and speculated such an alternating pattern would hold for all magic angles.

We also pointed out the intricate relation between the zeromode wave function and the quantum Hall wave functions. As guaranteed by intravalley inversion symmetry, the zero-mode wave function has an internal spinor structure, and in fact each component can be regarded as a product of a quantum Hall and an antiquantum Hall wave function, which guarantees the zero mode has the periodicity of a Bloch wave function. Interestingly, there are an increasing number of zeros occurring in each component at higher magic angles.

In the end, we discussed the implications of our results to realistic systems and observable phenomena. First, these zeros can be detected as charge minima in real space by scanning tunneling spectroscopy. Second, the increasing number of zeros present in the zero-mode wave function resembles the increasing number of zeros present in higher Landau level wave functions. Motivated by this observation, we anticipate higher Landau level physics will occur at the second and higher magic angles. Moreover, we noticed the phase circulation of the flat-band wave functions at higher magic angles, and anticipate phenomena related to magnetization. We leave more detailed studies on higher magic angles as future work. We notice an earlier work on low twist angle physics in Ref. [83].

Last but not least, it is well known that on a compact manifold, a U(1) magnetic field is subject to a Dirac quantization condition [84]. Our identification of zero-mode wave functions with two quantum Hall wave functions may also shed light on the non-Abelian quantization condition [85-87], where a semi-classical analysis was done recently in Ref. [88]).

Note added. Recently, we noticed the "unitary particlehole" symmetry occurring in Ref. [43], which is similar but distinct from our intravalley inversion symmetry in Eq. (15); we contrast the difference between the two in the paragraphs under Eq. (18). We also noticed a relevant work on the chiral model, Ref. [89], that appeared recently.

\section{ACKNOWLEDGMENTS}

The Flatiron Institute is a division of the Simons Foundation. This work was partially supported by the Air Force Office of Scientific Research under Grant No. FA9550-20-10260 (J.C.).

\section{APPENDIX A: MODEL HAMILTONIANS AND UNITARY TRANSFORMATIONS}

\section{Lattices}

We start with setting up the notation of moire lattice. As mentioned in the main text, we denote the two-dimensional lattice vectors as $\boldsymbol{a}_{i=1,2}^{a=x, y}$. The area of unit cell is defined to be $2 \pi S$ :

$$
2 \pi S \equiv\left|\boldsymbol{a}_{1} \times \boldsymbol{a}_{2}\right|=\left|\epsilon_{a b} \boldsymbol{a}_{1}^{a} \boldsymbol{a}_{2}^{b}\right|,
$$

where $\epsilon_{x y}=-\epsilon_{y x}=1$ is the antisymmetric symbol. The reciprocal basis vectors are

$$
\boldsymbol{b}_{a}^{i}=\epsilon^{i j} \epsilon_{a b} \boldsymbol{a}_{j}^{b} / S
$$

As will be shown in Appendix $\mathrm{C}, \sqrt{S}$ defines an effective magnetic length. We set $\sqrt{S}=1$ throughout this work.

Graphene contains A and B sites. As shown in Fig. 2 of the main text, the Dirac points $\boldsymbol{K} / \boldsymbol{K}^{\prime}$ and $A / B$ sites are located at

$$
\begin{array}{ll}
\boldsymbol{K}=\frac{-2 \boldsymbol{b}_{1}+\boldsymbol{b}_{2}}{3}, & \boldsymbol{K}^{\prime}=\frac{2 \boldsymbol{b}_{2}-\boldsymbol{b}_{1}}{3}, \\
\boldsymbol{r}_{A}=\frac{\boldsymbol{a}_{1}+2 \boldsymbol{a}_{2}}{3}, & \boldsymbol{r}_{B}=\frac{2 \boldsymbol{a}_{1}+\boldsymbol{a}_{2}}{3} .
\end{array}
$$

We use $\boldsymbol{r}_{0}$ for $\boldsymbol{r}_{A}$ throughout this work. 


\section{Unitary transformations}

In Sec. II, we described the Bistritzer-MacDonald Hamiltonian Eq. (3) and the chiral model Eq. (8) of a single valley. They are written in the nonchiral $\Psi_{\mathrm{BM}}$ and the chiral basis $\Psi_{c}$ respectively, see Eqs. (4) and (9). In this section, following Ref. [52], we work out the details of the unitary transformation between the two bases. We start with the continuum model Eq. (3), and perform a gauge transformation to remove the momentum shift on the diagonal. The Hamiltonian is transformed to be

$$
\begin{aligned}
H_{\mathrm{BM}} & =M_{T}\left(\begin{array}{cc}
-i v_{0} \boldsymbol{\sigma}_{+\theta / 2} \cdot \nabla & T(\boldsymbol{r}) \\
T^{\dagger}(\boldsymbol{r}) & -i v_{0} \boldsymbol{\sigma}_{-\theta / 2} \cdot \nabla
\end{array}\right) M_{T}^{\dagger}, \\
M_{T} & =\operatorname{diag}\left(e^{i \boldsymbol{K}_{+}^{b} \cdot \boldsymbol{r}}, e^{i \boldsymbol{K}_{+}^{t} \cdot \boldsymbol{r}}\right),
\end{aligned}
$$

where $T(\boldsymbol{r})$ is given in Eq. (6). Then, we remove the diagonal $\theta$ dependence by rotation:

$$
H_{\mathrm{BM}}=\left(M_{T} M_{\theta}\right) H_{\mathrm{cBM}}\left(M_{T} M_{\theta}\right)^{\dagger},
$$

where

$$
\begin{aligned}
H_{\mathrm{cBM}} & =\left(\begin{array}{cc}
-i v_{0} \boldsymbol{\sigma} \cdot \nabla & T(\boldsymbol{r}) \\
T^{\dagger}(\boldsymbol{r}) & -i v_{0} \boldsymbol{\sigma} \cdot \nabla
\end{array}\right), \\
M_{\theta} & =\operatorname{diag}\left(e^{-\frac{i \theta}{4} \sigma_{z}}, e^{\frac{i \theta}{4} \sigma_{z}}\right) .
\end{aligned}
$$

The matrix $H_{\mathrm{cBM}}$ is the chiral Hamiltonian organized in basis $\left(\phi_{b}, \chi_{b}, \phi_{t}, \chi_{t}\right)^{T}$ where $\phi$ and $\chi$ represent the A and B sublattices, respectively, and $b / t$ represent the bottom and top layer components. More explicitly,

$$
H_{\mathrm{cBM}}=\sqrt{2} v_{0}\left(\begin{array}{cccc}
0 & -i \partial & 0 & \alpha U_{-\phi} \\
-i \bar{\partial} & 0 & \alpha U_{\phi} & 0 \\
0 & \alpha U_{\phi}^{*} & 0 & -i \partial \\
\alpha U_{-\phi}^{*} & 0 & -i \bar{\partial} & 0
\end{array}\right) .
$$

where $U_{\phi}$ is defined in Eq. (13). Transforming into the chiral basis Eq. (9), we obtain Eq. (8):

$$
\begin{aligned}
H_{\mathrm{cBM}} & =v_{0}\left(\begin{array}{cc}
0 & \mathcal{D} \\
\mathcal{D}^{\dagger} & 0
\end{array}\right), \quad \mathcal{D}^{\dagger}=\sqrt{2}\left(\begin{array}{cc}
-i \bar{\partial} & \alpha U_{\phi} \\
\alpha U_{-\phi}^{*} & -i \bar{\partial}
\end{array}\right), \\
\mathcal{D} & =\sqrt{2}\left(\begin{array}{cc}
-i \partial & \alpha U_{-\phi} \\
\alpha U_{\phi}^{*} & -i \partial
\end{array}\right) .
\end{aligned}
$$

The unitary transformation Eq. (10) can be easily worked out from matrices $M_{T}, M_{\theta}$ and the basis shuffling. As defined in the main text, we denote the rotated graphene Dirac points as $\boldsymbol{K}_{+}^{b / t}$, and denote the moire Dirac points as $\boldsymbol{K}^{\mathrm{s}}=\boldsymbol{K}_{+}^{b}-\boldsymbol{K}_{+}^{\Gamma}$ and $\boldsymbol{K}^{\prime}=\boldsymbol{K}_{+}^{t}-\boldsymbol{K}_{+}^{\Gamma}$, where $\boldsymbol{K}_{+}^{\Gamma}$ is the moire Brillouin zone center. We have also shifted the Bloch momentum of the chiral basis Eq. (9) to center at the moire Gamma point. Its Bloch translation symmetry can be also worked out easily as shown in Eq. (11).

\section{APPENDIX B: HOW $\mathcal{C}_{2} \mathcal{T}$ SYMMETRY CONSTRAINS THE CHIRAL HAMILTONIAN}

We have written the inter-layer coupling matrix in real space as Eq. (3). We now discuss the action of $\mathcal{C}_{2} \mathcal{T}$, which complex conjugates and exchanges the two sublattices. The diagonal blocks in Eq. (3) are invariant under this transformation. We now consider the off-diagonal tunneling terms $H_{\mathrm{BM}}^{\text {tun }}$.
Its transformation under $\mathcal{C}_{2} \mathcal{T}$ reads

$$
\begin{aligned}
H_{\mathrm{BM}}^{\mathrm{tun}} & \stackrel{\mathcal{C}_{2} \mathcal{T}}{\longrightarrow} \int d \boldsymbol{r} \Psi^{\dagger}(-\boldsymbol{r})\left(\begin{array}{cc}
0 & \sigma_{x} T(\boldsymbol{r}) \sigma_{x} \\
\sigma_{x} T^{\dagger}(\boldsymbol{r}) \sigma_{x} & 0
\end{array}\right)^{*} \Psi(-\boldsymbol{r}) \\
& =\int d \boldsymbol{r} \Psi^{\dagger}(\boldsymbol{r})\left(\begin{array}{cc}
0 & \sigma_{x} T(-\boldsymbol{r}) \sigma_{x} \\
\sigma_{x} T^{\dagger}(-\boldsymbol{r}) \sigma_{x} & 0
\end{array}\right)^{*} \Psi(\boldsymbol{r}),
\end{aligned}
$$

where, same as the main text, the Pauli matrices $\sigma$ act on sublattice space. By virtue of being invariant under $\mathcal{C}_{2} \mathcal{T}$, it follows that

$$
T(\boldsymbol{r})=\sigma_{x} T^{*}(-\boldsymbol{r}) \sigma_{x},
$$

or, element by element:

$$
T_{A A}(\boldsymbol{r})=T_{B B}^{*}(-\boldsymbol{r}), \quad T_{A B}(\boldsymbol{r})=T_{B A}^{*}(-\boldsymbol{r}) .
$$

If we rotate to the chiral basis $\Psi_{c}$ Eq. (9), the tunneling terms enter in the following way:

$$
\mathcal{H}_{\mathrm{cBM}}(\boldsymbol{r})=\left(\begin{array}{cc}
T_{A}^{\mathrm{diag}}(\boldsymbol{r}) & \mathcal{D}(\boldsymbol{r}) \\
\mathcal{D}^{\dagger}(\boldsymbol{r}) & T_{B}^{\mathrm{diag}}(\boldsymbol{r})
\end{array}\right),
$$

where the diagonal blocks are

$$
\begin{aligned}
& T_{A}^{\text {diag }}(\boldsymbol{r})=\left(\begin{array}{ll}
T_{A A}^{*}(\boldsymbol{r}) & T_{A A}(\boldsymbol{r})
\end{array}\right), \\
& T_{B}^{\text {diag }}(\boldsymbol{r})=\left(\begin{array}{ll}
T_{B B}^{*}(\boldsymbol{r}) & T_{B B}(\boldsymbol{r})
\end{array}\right),
\end{aligned}
$$

and the off-diagonal blocks are

$$
\begin{gathered}
\mathcal{D}(\boldsymbol{r})=\left(\begin{array}{cc}
-\sqrt{2} i \partial & T_{B A}^{*}(\boldsymbol{r}) \\
T_{A B}(\boldsymbol{r}) & -\sqrt{2} i \partial
\end{array}\right), \\
\mathcal{D}^{\dagger}(\boldsymbol{r})=\left(\begin{array}{cc}
-\sqrt{2} i \bar{\partial} & T_{A B}^{*}(\boldsymbol{r}) \\
T_{B A}(\boldsymbol{r}) & -\sqrt{2} i \bar{\partial}
\end{array}\right) .
\end{gathered}
$$

Using the action of $\mathcal{C}_{2} \mathcal{T}$ in Eq. (B2), these can be written in terms of only one complex parameter $T_{A B}$ :

$$
\begin{gathered}
\mathcal{D}(\boldsymbol{r})=\left(\begin{array}{cc}
-i \sqrt{2} \partial & T_{A B}(-\boldsymbol{r}) \\
T_{A B}(\boldsymbol{r}) & -\sqrt{2} i \partial
\end{array}\right), \\
\mathcal{D}^{\dagger}(\boldsymbol{r})=\left(\begin{array}{cc}
-\sqrt{2} i \bar{\partial} & T_{A B}^{*}(\boldsymbol{r}) \\
T_{A B}^{*}(-\boldsymbol{r}) & -\sqrt{2} i \bar{\partial}
\end{array}\right),
\end{gathered}
$$

which satisfy our Lemma 1:

$$
\tau_{y} \mathcal{D}^{\dagger}(\boldsymbol{r}) \tau_{y}=-\mathcal{D}^{\dagger}(-\boldsymbol{r}), \quad \tau_{y} \mathcal{D}(\boldsymbol{r}) \tau_{y}=-\mathcal{D}(-\boldsymbol{r}),
$$

where the Pauli matrices $\tau$ act on the layer index.

In this basis, the chiral matrix $\sigma_{z}$ that anticommutes with the Hamiltonian enforces $T_{A A}(\boldsymbol{r})=T_{B B}(\boldsymbol{r})=0$. Notice that Eq. (B7) also requires linearized Dirac fermion; a quadratic term in the dispersion destroys it. Note that a quadratic term in the dispersion also destroys the exact flat band of the chiral model. We hence demonstrated that for chiral models with linearized Dirac fermion, intravalley inversion follows from $\mathcal{C}_{2} \mathcal{T}$ symmetry.

\section{APPENDIX C: QUANTUM HALL WAVE FUNCTION}

In this section, we review the quantum Hall wave function that is frequently used in the main text. We start with discussing magnetic translation symmetry and quasiperiodic elliptic functions. 


\section{Magnetic translation symmetry}

Since the lowest Landau level wave functions are usually written in terms of holomorphic functions, we start by setting up a notation for complex coordinates. Complex structures $\omega_{a=x, y}$ and $\omega_{a=x, y}^{*}$ define a one-to-one mapping from twodimensional affine space to the complex plane. We represent the metric and the antisymmetric tensor as

$$
g_{a b}=\omega_{a}^{*} \omega_{b}+\omega_{a} \omega_{b}^{*}, \quad i \epsilon_{a b}=\omega_{a}^{*} \omega_{b}-\omega_{a} \omega_{b}^{*} .
$$

They have the properties: $\omega^{a}=g^{a b} \omega_{b}, \omega^{a} \omega_{a}=0$, and $\omega^{a} \omega_{a}^{*}=1$. The complex vectors are defined by contracting complex structure with vectors $A \equiv \omega_{a} \boldsymbol{A}^{a}$, and complex covectors as $B \equiv \omega^{a} \boldsymbol{B}_{a}$. To distinguish with vectors, complex vectors are unbold. In terms of complex coordinates, the inner product and cross product are respectively $\boldsymbol{A} \cdot \boldsymbol{B} \equiv \boldsymbol{A}_{a} \boldsymbol{B}^{a}=$ $A B^{*}+A^{*} B$, and $\boldsymbol{A} \times \boldsymbol{B} \equiv \epsilon_{a b} \boldsymbol{A}^{a} \boldsymbol{B}^{b}=-i\left(A^{*} B-A B^{*}\right)$. In this work, we took $\omega_{x}=1 / \sqrt{2}$ and $\omega_{y}=i / \sqrt{2}$.

The quantum Hall system describes two-dimensional interacting or noninteracting electrons in a perpendicular magnetic field. In a magnetic field, the electron's coordinate is factorized into the center of its cyclotron motion i.e., guiding center $\boldsymbol{R}$, and the radius, i.e., Landau orbits $\overline{\boldsymbol{R}}$ :

$$
\boldsymbol{r}=\boldsymbol{R}+\overline{\boldsymbol{R}},
$$

where $\boldsymbol{R}$ commutes with $\overline{\boldsymbol{R}}$, but their individual components are noncommutative:

$$
\left[\boldsymbol{R}^{a}, \boldsymbol{R}^{b}\right]=-i \epsilon^{a b} l_{B}^{2}, \quad\left[\overline{\boldsymbol{R}}^{a}, \overline{\boldsymbol{R}}^{b}\right]=i \epsilon^{a b} l_{B}^{2} .
$$

In our case the area of unit cell $S$ plays the same role as magnetic length squared $l_{B}^{2}=\hbar /|e B|$ where $e, B$ are electron charge and magnetic field strength. When projected into a single Landau level, an electron is fully described by the noncommutative $\boldsymbol{R}$ degrees of freedom. The magnetic translation operator is defined as the following one:

$$
t(\boldsymbol{d}) \equiv \exp (i \boldsymbol{d} \times \boldsymbol{R}),
$$

which translates the guiding center $\boldsymbol{R}$ by distance $\boldsymbol{d}$. The magnetic translation algebra is

$$
t\left(\boldsymbol{d}_{1}\right) t\left(\boldsymbol{d}_{2}\right)=t\left(\boldsymbol{d}_{2}\right) t\left(\boldsymbol{d}_{1}\right) e^{i \boldsymbol{d}_{1} \times \boldsymbol{d}_{2}}=t\left(\boldsymbol{d}_{1}+\boldsymbol{d}_{2}\right) e^{\frac{i}{2} \boldsymbol{d}_{1} \times \boldsymbol{d}_{2}} .
$$

Due to the single value of wave function, any legal wave function must transform back to itself after a periodic translation. So we have the boundary condition

$$
t(\boldsymbol{a}) \psi=e^{i \phi_{a}} \psi
$$

where $\boldsymbol{a} \in \mathbb{A}$ is a lattice vector. From now on we define the whole lattice as $\mathbb{A} \equiv\left\{m \boldsymbol{a}_{1}+n \boldsymbol{a}_{2} \mid m, n \in \mathbb{Z}\right\}$. The phase factor $\phi_{a}$ effectively measures the fraction of flux inside the torus. The wave functions that satisfy Eq. (C5) are written in terms of elliptic functions. One choice of elliptic function is the Jacobi theta function [60]. Recently it was also found that the "modified Weierstrass sigma function" is another choice [56,57]. Compared with Jacobi theta function, Weierstrass sigma function has the advantage of being modular invariant.

\section{Modified Weierstrass sigma function}

The modified Weierstrass sigma function [56,57] $\sigma(z)$ is defined as

$$
\sigma(z)=\tilde{\sigma}(z) e^{-\frac{1}{2} \bar{G}(\mathbb{A}) z^{2}},
$$

i.e., a product of the standard Weierstrass sigma function $\tilde{\sigma}(z)$ and a holomorphic factor $e^{-\frac{1}{2} \bar{G}(\mathbb{A}) z^{2}}$, where as will be explained soon the "almost modular form" $\bar{G}(\mathbb{A})$ is a modular independent $c$-number constant that vanishes for square and hexagonal torus. We now introduce the $\bar{G}(\mathbb{A})$, and discuss the quasiperiodic property of $\sigma(z)$.

The standard Weierstrass sigma function $\tilde{\sigma}(z)$ has a product series expansion (which is also a fast converging form for numerics),

$$
\tilde{\sigma}(z) \equiv z \prod_{a \in A_{m n} \backslash\{0\}}\left(1-\frac{z}{a}\right) e^{\frac{z}{a}+\frac{1}{2} \frac{z^{2}}{a^{2}}},
$$

where as defined above, $\mathbb{A}$ means the set of lattice points. Clearly, it is modular invariant. It is also quasiperiodic,

$$
\tilde{\sigma}\left(z+a_{i}\right)=-e^{2 \tilde{\eta}_{i}\left(z+a_{i} / 2\right)} \tilde{\sigma}(z), \quad i=1,2,
$$

where $\tilde{\eta}_{i}$ is the standard zeta function evaluated at half period, which is related to the $k=1$ Eisenstein series $G_{2}\left(a_{i}\right), i=1,2$,

$$
\tilde{\eta}_{i}=G_{2}\left(a_{i}\right) a_{i} / 2 \text {. }
$$

The Eisenstein series $G_{2}\left(a_{i}\right)$ has a highly convergent formula

$$
G_{2}\left(a_{i}\right)=\frac{2 \pi^{2}}{a_{i}^{2}}\left(\frac{1}{6}+\sum_{n=1}^{\infty} \frac{1}{\sin ^{2}\left(n \pi \frac{a_{j \neq i}}{a_{i}}\right)}\right) .
$$

The $\tilde{\eta}_{i}$ in addition obey a relation that defines chirality,

$$
\tilde{\eta}_{1} a_{2}-\tilde{\eta}_{2} a_{1}=\frac{1}{2 N_{\phi}}\left(a_{1}^{*} a_{2}-a_{1} a_{2}^{*}\right)=i \pi .
$$

In our case, the magnetic flux quanta of a unit cell is one, so $N_{\phi}=1$. The (C8) and (C10) suggests a modular independent quantity called "almost modular form,"

$$
\bar{G}(\mathbb{A}) \equiv G_{2}\left(a_{i}\right)-\frac{1}{N_{\phi}} \frac{a_{i}^{*}}{a_{i}} .
$$

With these formulas in hand, we are ready to get the quasiperiodicity of $\sigma(z)$ :

$$
\sigma\left(z+a_{i}\right)=-e^{a_{i}^{*}\left(z+a_{i} / 2\right)} \sigma(z), \quad i=1,2 .
$$

Last but not least, the sigma function is odd under spatial inversion: $\sigma(-z)=-\sigma(z)$.

\section{Quantum Hall wave function}

The quantum Hall wave function is given in Eq. (31), which we copy below:

$$
\Phi_{\boldsymbol{k}}(\boldsymbol{r})=e^{z_{k}^{*} z} \sigma\left(z-z_{k}\right) e^{-\frac{1}{2}\left|z_{k}\right|^{2}} e^{-\frac{1}{2}|z|^{2}} .
$$

It has a single zero located at $\boldsymbol{r}_{\boldsymbol{k}}^{a}=\boldsymbol{r}_{0}^{a}+\epsilon^{a b}(\boldsymbol{k}-\boldsymbol{K})_{b}$ in each unit cell, with $\boldsymbol{r}_{0}$ defined in Eq. (24). Mapping to the complex plane, the zero occurs at $z_{k}$ and its translated counterparts, where $z_{k}$ is

$$
z_{k}=\omega_{a}\left(\boldsymbol{r}_{0}^{a}-\epsilon^{a b} \boldsymbol{K}_{b}\right)+\omega_{a} \epsilon^{a b} \boldsymbol{k}_{b}=-i k,
$$




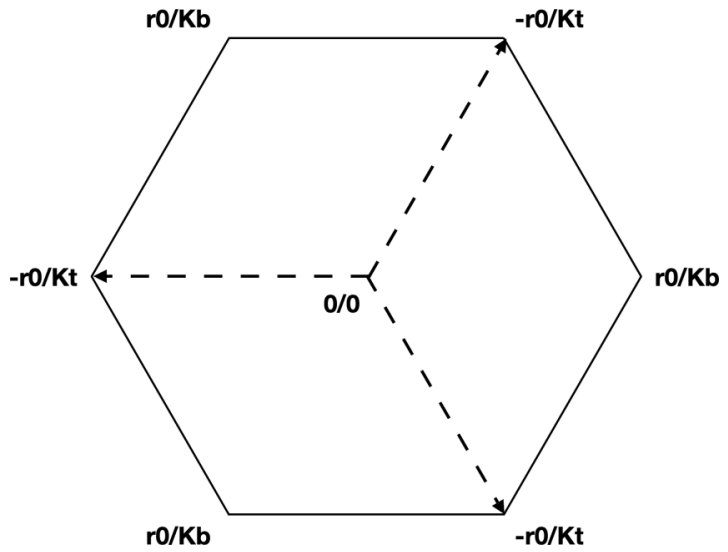

FIG. 7. The one-to-one mapping between $\boldsymbol{k}$ and $\boldsymbol{r}_{\boldsymbol{k}}$, where the first and second letter are $\boldsymbol{r}_{\boldsymbol{k}}$ and $\boldsymbol{k}$, respectively. The figure is constructed by rotating the moire Brillouin zone by 90 degrees and overlaps with the real-space unit cell, precisely because of the mathematical relation $\boldsymbol{r}_{\boldsymbol{k}}^{a}=\boldsymbol{r}_{0}^{a}+\epsilon^{a b}(\boldsymbol{k}-\boldsymbol{K})_{b}$. All points in the diagram are illustrated modulo lattice vectors.

where the first term is zero following from Eqs. (A2) and (A3). We used Eq. (C1) to derive the second term.

Since $\Phi_{\boldsymbol{k}}$ is not a Bloch function, the "Bloch vector" $\boldsymbol{k}$ here should be understood as labeling the magnetic translation boundary condition Eq. (C5): $t\left(\boldsymbol{a}_{1,2}\right) \Phi_{\boldsymbol{k}}=-e^{i \boldsymbol{k} \cdot \boldsymbol{a}_{1,2}} \Phi_{\boldsymbol{k}}$. For a quantum Hall wave function, its zero moves linearly with the boundary condition $\boldsymbol{k}$, reflecting the fact of Chern number $\mathcal{C}=1$ [62]. The following diagram Fig. 7 is helpful to quickly figure out $\boldsymbol{r}_{\boldsymbol{k}}$ given the Bloch momentum $\boldsymbol{k}$.

Using results derived in the last section, it is easy to find the quasiperiodic boundary condition $\Phi_{k}$ satisfies in real and reciprocal space. With $i=1,2$, they are

$$
\begin{gathered}
\Phi_{\boldsymbol{k}}\left(\boldsymbol{r}+\boldsymbol{a}_{i}\right)=-e^{\frac{i}{2} \boldsymbol{a}_{i} \times \boldsymbol{r}} e^{i \boldsymbol{r}_{\boldsymbol{k}} \times \boldsymbol{a}_{i}} \Phi_{\boldsymbol{k}}(\boldsymbol{r}), \\
\Phi_{\boldsymbol{k}+\boldsymbol{b}_{i}}(\boldsymbol{r})=-e^{\frac{i}{2} \boldsymbol{b}_{i} \cdot \boldsymbol{r}_{\boldsymbol{k}}} \Phi_{\boldsymbol{k}}(\boldsymbol{r}) .
\end{gathered}
$$

The nontrivial phase factors above cannot be removed by a smooth, global, gauge transformation, which reflects the fact that $\Phi_{k}$ has a nontrivial Chern number. Technically, these boundary conditions allow one to restrict the discussion to the unit cell and the first Brillouin zone. From now on, we denote $\boldsymbol{k}$ as Bloch momentum inside the first Brillouin zone.

It is straightforward to see how inversion acts on quantum Hall wave functions from Eq. (C13):

$$
\Phi_{k}(\boldsymbol{r})=-\Phi_{-k}(-\boldsymbol{r}) .
$$

We finish this section by showing the boundary condition of $\mathcal{G}(\boldsymbol{r})$, which follows straightforwardly from the periodicity of the zero-mode wave function in Eq. (11) and the quantum Hall wave function in Eq. (C14):

$$
\mathcal{G}\left(\boldsymbol{r}+\boldsymbol{a}_{i=1,2}\right)=-\mathcal{G}(\boldsymbol{r}) \times e^{-\frac{i}{2} \boldsymbol{a}_{i} \times \boldsymbol{r}} e^{i \boldsymbol{q}_{0} \cdot \boldsymbol{a}_{i}} .
$$

\section{APPENDIX D: ANALYTICAL ARGUMENT FOR THE NODAL STRUCTURE}

The alternating parities and the increasing number of zeros we observed in the chiral model shares many similarities as the simple harmonic oscillator. In this section, we provide an argument for the zero structure and inversion patterns by making an analogy to simple one-dimensional harmonic oscillators. Specifically, since the additional zeros that occur at higher magic angles occur along a reflection symmetric line, we reduce the zero-mode equation to a one variable ordinary differential equation on that line. We can then compare to a harmonic oscillator in one dimension.

The one-dimensional harmonic oscillator is described by the Hamiltonian:

$$
H=\frac{\hat{p}^{2}}{2 m}+\frac{1}{2}\left(m \omega^{2}\right) x^{2},
$$

whose $n_{t h}$ eigenstate $\phi_{n}(x)$ satisfies the eigenequation:

$$
-\frac{\hbar^{2}}{2 m} \frac{d^{2} \phi_{n}}{d x^{2}}+\frac{1}{2}\left(m \omega^{2}\right) x^{2} \phi_{n}^{2}=E_{n} \phi_{n},
$$

which can be transformed into the standard Sturm-Liouville form, with dimensionless parameters $\alpha \equiv \sqrt{\hbar /(m \omega)}, \epsilon \equiv$ $E /(\hbar \omega / 2)$ and $u \equiv x / \alpha$ :

$$
\frac{d}{d u}\left[p(u) \frac{d \phi_{n}(u)}{d u}\right]+(q(u)+\epsilon \omega(u)) \phi_{n}(u)=0,
$$

where

$$
p(u)=1, \quad q(u)=-u^{2}, \quad \omega(u)=1 .
$$

The normalizable solutions of Eq. (D3) are given by,

$$
\phi_{n}(u)=N_{n} \mathcal{H}_{n}(u) e^{-\frac{1}{2} u^{2}},
$$

where $N_{n}=\left(\frac{m \omega}{\pi \hbar}\right)^{\frac{1}{4}}\left(2^{n} n !\right)^{-\frac{1}{2}}$ is the normalization factor and $\mathcal{H}_{n}$ is the $n_{t h}$ Hermite polynomial. Therefore we see that for the harmonic oscillator, the number of zeros of the $n$th excited eigenstate is $n$, and the parity of the $n_{t h}$ eigenstate $\psi_{n}$ alternates as $(-1)^{n}$. Such oscillatory behavior is a generic feature for Sturm-Liouville type differential equations Eq. (D3) on the interval where $p(u)$ and $\omega(u)$ are positive [65].

We have observed a similar alternating parity and increasing number of zeros of eigenstates at higher magic angles in the chiral model, as discussed in Secs. III B and V C. The problem of the chiral twisted bilayer graphene model is more difficult. One reason is that it is a two-variable differential equation. To make progress, we utilize the symmetry of the problem to reduce the problem to one variable.

We starting by reviewing the zero-mode equation, and see how symmetry helps reduce the dimension of the problem. We first recall the zero-mode equation from Eqs. (1) and (12):

$$
-i \bar{\partial}\left(i \mathcal{G}(\boldsymbol{r}) \Phi_{\boldsymbol{k}}(\boldsymbol{r})\right)=-\eta \alpha U_{\phi}(\boldsymbol{r}) \mathcal{G}(-\boldsymbol{r}) \Phi_{\boldsymbol{k}}(\boldsymbol{r}) .
$$

By using the lowest Landau level condition that the quantum Hall wave function $\Phi_{k}$ satisfies

$$
\bar{\partial} \Phi_{k}=-\frac{z}{2} \Phi_{k},
$$

we arrive at the zero-mode equation that the function $\mathcal{G}(\boldsymbol{r})$ must satisfy

$$
\left(\bar{\partial}-\frac{z}{2}\right) \mathcal{G}(\boldsymbol{r})+\eta \alpha U_{\phi}(\boldsymbol{r}) \mathcal{G}(-\boldsymbol{r})=0,
$$

which is subject to the boundary condition Eq. (C17). 
We note that due to the mirror symmetry $\mathcal{M}_{y}$ of the problem, both $\mathcal{G}(x, y)$ and $\mathcal{G}^{*}(x,-y)$ are zero-mode solutions of Eq. (D8). By utilizing the global U(1) phase degree of freedom of wave function, one can always choose $\mathcal{G}(\boldsymbol{r}=\mathbf{0})$ to be a purely real number, thereby constraining $\mathcal{G}(x, 0)$ to be a real function. We have already used this property for $\rho(\boldsymbol{r})$ in Sec. VC, and plotted its real and imaginary parts on the reflection symmetric line in Fig. 4.

Here we denote the real and imaginary parts of $\mathcal{G}(x, 0)$ as $\mathcal{R}(x)$ and $\mathcal{I}(x)$ respectively. Although the imaginary part vanishes identically at $y=0$, its $y$-direction derivative $\left(\partial_{y} \mathcal{I}\right)(x) \equiv$ $\left.\partial_{y} \mathcal{I}(x, y)\right|_{y=0}$ does not. We end up with the following:

$$
\mathcal{I}(x)=0, \quad \mathcal{R}(x) \neq 0, \quad\left(\partial_{y} \mathcal{I}\right)(x) \neq 0 .
$$

The zero-mode equation Eq. (D8) is now rewritten as

$$
\partial_{x} \mathcal{R}(x)-\left(\partial_{y} \mathcal{I}\right)(x)-\frac{x}{2} \mathcal{R}(x)+\eta \alpha U_{\phi}(x) \mathcal{R}(-x)=0,
$$

subject to the boundary condition Eq. (C17) which, when reduced to the $y=0$ line, becomes

$$
\begin{aligned}
\mathcal{R}(x+\sqrt{3} a) & =-\mathcal{R}(x), \\
\left(\partial_{y} \mathcal{I}\right)(x+\sqrt{3} a) & =-\left(\partial_{y} \mathcal{I}\right)(x)+\frac{\sqrt{3} a}{2} \mathcal{R}(x),
\end{aligned}
$$

where $a$ is the length of the moire primitive lattice vectors. In the unit $S=1$, we have been using, its value is $a^{2}=4 \pi / \sqrt{3}$.

The derivation so far is exact. The differential equation Eq. (D10) and its boundary conditions Eq. (D11) contain the full information of the nodes in the problem. The difficulty of solving Eq. (D10) is that it is a two-variable differential equation. To make progress, we now do approximation on $\left(\partial_{y} \mathcal{I}\right)$ to eliminate one variable.

It is interesting to observe that $-\frac{x}{2} \mathcal{R}(x)$ satisfies the same boundary condition as $\left(\partial_{y} \mathcal{I}\right)(x)$. In the following, we will approximate

$$
\left(\partial_{x} \mathcal{I}\right)(x) \approx-\frac{x}{2} \mathcal{R}(x)
$$

Under this assumption, the differential equation simplifies dramatically, and becomes a one-variable ordinary differential equation:

$$
\frac{d}{d x} \mathcal{R}(x)+\eta \alpha U_{\phi}(x) \mathcal{R}(-x)=0,
$$

which can be rewritten into a second-order form:

$$
\begin{aligned}
-\frac{d}{d x}\left(\frac{1}{U_{\phi}(x)} \frac{d \mathcal{R}(x)}{d x}\right) & =\alpha^{2} U_{\phi}(-x) \mathcal{R}(x) . \\
\mathcal{R}(x+\sqrt{3} a) & =-\mathcal{R}(x) .
\end{aligned}
$$

Hence we have brought the zero-mode equation on the reflection symmetric line into the Sturm-Liouville form Eq. (D3) under the approximation shown in Eq. (D12).

Suppose we have two solutions $\mathcal{R}_{1,2}$ of Eq. (D14), which corresponds to two magic angles $\alpha_{1,2}$, with $\alpha_{1}<\alpha_{2}$. From Eq. (D14), we deduce that

$$
\left[U_{\phi}^{-1}\left(\mathcal{R}_{1} \mathcal{R}_{2}^{\prime}-\mathcal{R}_{1}^{\prime} \mathcal{R}_{2}\right)\right]^{\prime}=\left(\alpha_{1}^{2}-\alpha_{2}^{2}\right) U_{\phi}(-x) \mathcal{R}_{1} \mathcal{R}_{2},
$$

where we have implicitly suppressed the argument $x$ in $U_{\phi}^{-1}$, $\mathcal{R}_{12}$ and their derivatives. Now, consider a region spanned $\left[x_{a}, x_{b}\right]$. The integration of the above equation in this region yields

$$
\begin{aligned}
& {\left.\left[U_{\phi}^{-1}(x)\left(\mathcal{R}_{1}(x) \mathcal{R}_{2}^{\prime}(x)-\mathcal{R}_{1}^{\prime}(x) \mathcal{R}_{2}(x)\right)\right]\right|_{x_{a}} ^{x_{b}}} \\
& \quad=\left(\alpha_{1}^{2}-\alpha_{2}^{2}\right) \int_{x_{a}}^{x_{b}} d \zeta U_{\phi}(-\zeta) \mathcal{R}_{1}(\zeta) \mathcal{R}_{2}(\zeta) .
\end{aligned}
$$

It then follows from the theory of differential equations [65], in the parameter region $x \in\left[x_{a}, x_{b}\right]$ that $U_{\phi}( \pm x)>$ 0 or $U_{\phi}( \pm x)<0$, the nodes of two consecutive solutions must oscillate; otherwise it leads to contradiction with Eq. (D15). We emphasize that our argument is based on the assumption Eq. (D12), and we can only argue for the node oscillation in the regions where $U_{\phi}( \pm x)$ are both positive or negative. This argument shows that in general, there should be more zeros at higher magic angles.
[1] R. Bistritzer and A. H. MacDonald, Moiré bands in twisted double-layer graphene, Proc. Natl. Acad. Sci. 108, 12233 (2011).

[2] J. M. B. Lopes dos Santos, N. M. R. Peres, and A. H. Castro Neto, Continuum model of the twisted graphene bilayer, Phys. Rev. B 86, 155449 (2012).

[3] J. M. B. Lopes dos Santos, N. M. R. Peres, and A. H. Castro Neto, Graphene Bilayer with a Twist: Electronic Structure, Phys. Rev. Lett. 99, 256802 (2007).

[4] E. J. Mele, Commensuration and interlayer coherence in twisted bilayer graphene, Phys. Rev. B 81, 161405(R) (2010).

[5] Y. Cao, V. Fatemi, S. Fang, K. Watanabe, T. Taniguchi, E. Kaxiras, and P. Jarillo-Herrero, Unconventional superconductivity in magic-angle graphene superlattices, Nature (London) 556, 43 (2018).

[6] Y. Cao, V. Fatemi, A. Demir, S. Fang, S. L. Tomarken, J. Y. Luo, J. D. Sanchez-Yamagishi, K. Watanabe, T. Taniguchi, E. Kaxiras, R. C. Ashoori, and P. Jarillo-Herrero, Correlated insulator behavior at half-filling in magic-angle graphene superlattices, Nature (London) 556, 80 (2018).

[7] M. Serlin, C. L. Tschirhart, H. Polshyn, Y. Zhang, J. Zhu, K. Watanabe, T. Taniguchi, L. Balents, and A. F. Young, Intrinsic quantized anomalous Hall effect in a moiré heterostructure, Science 367, 900 (2020).

[8] A. L. Sharpe, E. J. Fox, A. W. Barnard, J. Finney, K. Watanabe, T. Taniguchi, M. A. Kastner, and D. GoldhaberGordon, Emergent ferromagnetism near three-quarters filling in twisted bilayer graphene, Science 365, 605 (2019).

[9] M. Yankowitz, S. Chen, H. Polshyn, Y. Zhang, K. Watanabe, T. Taniguchi, D. Graf, A. F. Young, and C. R. Dean, Tuning superconductivity in twisted bilayer graphene, Science 363, 1059 (2019).

[10] H. Polshyn, M. Yankowitz, S. Chen, Y. Zhang, K. Watanabe, T. Taniguchi, C. R. Dean, and A. F. Young, Large linear-intemperature resistivity in twisted bilayer graphene, Nat. Phys. 15, 1011 (2019). 
[11] Y. Saito, J. Ge, K. Watanabe, T. Taniguchi, and A. F. Young, Independent superconductors and correlated insulators in twisted bilayer graphene, Nat. Phys. 16, 926 (2020).

[12] Y. Tang, L. Li, T. Li, Y. Xu, S. Liu, K. Barmak, K. Watanabe, T. Taniguchi, A. H. MacDonald, J. Shan, and K. F. Mak, Simulation of Hubbard model physics in $\mathrm{WSe}_{2} / \mathrm{WS}_{2}$ moirésuperlattices, Nature (London) 579, 353 (2020).

[13] L. Balents, C. R. Dean, D. K. Efetov, and A. F. Young, Superconductivity and strong correlations in moiré flat bands, Nat. Phys. 16, 725 (2020).

[14] C. Xu and L. Balents, Topological Superconductivity in Twisted Multilayer Graphene, Phys. Rev. Lett. 121, 087001 (2018).

[15] Fengcheng Wu, A. H. MacDonald, and I. Martin, Theory of Phonon-Mediated Superconductivity in Twisted Bilayer Graphene, Phys. Rev. Lett. 121, 257001 (2018).

[16] B. Lian, Z. Wang, and B. A. Bernevig, Twisted Bilayer Graphene: A phonon-Driven Superconductor, Phys. Rev. Lett. 122, 257002 (2019).

[17] X. Hu, T. Hyart, D. I. Pikulin, and E. Rossi, Geometric and Conventional Contribution to the Superfluid Weight in Twisted Bilayer Graphene, Phys. Rev. Lett. 123, 237002 (2019).

[18] F. Xie, Z. Song, B. Lian, and B. A. Bernevig, TopologyBounded Superfluid Weight in Twisted Bilayer Graphene, Phys. Rev. Lett. 124, 167002 (2020).

[19] Fengcheng Wu and S. Das Sarma, Collective Excitations of Quantum Anomalous Hall Ferromagnets in Twisted Bilayer Graphene, Phys. Rev. Lett. 124, 046403 (2020).

[20] Y.-H. Zhang, D. Mao, Y. Cao, P. Jarillo-Herrero, and T. Senthil, Nearly flat Chern bands in moiré superlattices, Phys. Rev. B 99, 075127 (2019).

[21] Y. Gao, Y. Zhang, and D. Xiao, Tunable Layer Circular Photogalvanic Effect in Twisted Bilayers, Phys. Rev. Lett. 124, 077401 (2020).

[22] G. Li, A. Luican, J. M. B. Lopes dos Santos, A. H. Castro Neto, A. Reina, J. Kong, and E. Y. Andrei, Observation of van Hove singularities in twisted graphene layers, Nat. Phys. 6, 109 (2010).

[23] G. Trambly de Laissardière, D. Mayou, and L. Magaud, Localization of dirac electrons in rotated graphene bilayers, Nano Lett. 10, 804 (2010).

[24] L. Rademaker and P. Mellado, Charge-transfer insulation in twisted bilayer graphene, Phys. Rev. B 98, 235158 (2018).

[25] M. Xie and A. H. MacDonald, Nature of the Correlated Insulator States in Twisted Bilayer Graphene, Phys. Rev. Lett. 124, 097601 (2020).

[26] J. González and T. Stauber, Marginal Fermi Liquid in Twisted Bilayer Graphene, Phys. Rev. Lett. 124, 186801 (2020).

[27] Y. Saito, J. Ge, K. Watanabe, T. Taniguchi, E. Berg, and A. F. Young, Isospin Pomeranchuk effect and the entropy of collective excitations in twisted bilayer graphene, arXiv:2008.10830 [cond-mat.mes-hall].

[28] X. Lu, B. Lian, G. Chaudhary, B. A. Piot, G. Romagnoli, K. Watanabe, T. Taniguchi, M. Poggio, A. H. MacDonald, B. A. Bernevig, and D. K. Efetov, Fingerprints of Fragile Topology in the Hofstadter spectrum of Twisted Bilayer Graphene Close to the Second Magic Angle, arXiv:2006.13963 [cond-mat.meshall].

[29] E. Khalaf, N. Bultinck, A. Vishwanath, and M. P. Zaletel, Soft modes in magic angle twisted bilayer graphene, arXiv:2009.14827 [cond-mat.str-el].
[30] E. J. Mele, Band symmetries and singularities in twisted multilayer graphene, Phys. Rev. B 84, 235439 (2011).

[31] H. C. Po, L. Zou, A. Vishwanath, and T. Senthil, Origin of Mott Insulating Behavior and Superconductivity in Twisted Bilayer Graphene, Phys. Rev. X 8, 031089 (2018).

[32] L. Zou, H. C. Po, A. Vishwanath, and T. Senthil, Band structure of twisted bilayer graphene: Emergent symmetries, commensurate approximants, and wannier obstructions, Phys. Rev. B 98 085435 (2018).

[33] J. Kang and O. Vafek, Symmetry, Maximally Localized Wannier States, and a Low-Energy Model for Twisted Bilayer graphene Narrow Bands, Phys. Rev. X 8, 031088 (2018).

[34] M. Koshino, N. F. Q. Yuan, T. Koretsune, M. Ochi, K. Kuroki, and L. Fu, Maximally Localized Wannier Orbitals and the Extended Hubbard Model for Twisted Bilayer Graphene, Phys. Rev. X 8, 031087 (2018).

[35] Z. Song, Z. Wang, W. Shi, G. Li, C. Fang, and B. A. Bernevig, All Magic Angles in Twisted Bilayer Graphene are Topological, Phys. Rev. Lett. 123, 036401 (2019).

[36] H. Isobe, N. F. Q. Yuan, and L. Fu, Unconventional Superconductivity and Density Waves in Twisted Bilayer Graphene, Phys. Rev. X 8, 041041 (2018).

[37] H. C. Po, L. Zou, T. Senthil, and A. Vishwanath, Faithful tight-binding models and fragile topology of magic-angle bilayer graphene, Phys. Rev. B 99, 195455 (2019).

[38] S. Carr, S. Fang, H. C. Po, A. Vishwanath, and E. Kaxiras, Derivation of wannier orbitals and minimal-basis tight-binding hamiltonians for twisted bilayer graphene: First-principles approach, Phys. Rev. Research 1, 033072 (2019).

[39] O. Vafek and J. Kang, Towards the Hidden Symmetry in Coulomb Interacting Twisted Bilayer Graphene: Renormalization Group Approach, Phys. Rev. Lett. 125, 257602 (2020).

[40] J. Kang and O. Vafek, Strong Coupling Phases of Partially Filled Twisted Bilayer Graphene Narrow Bands, Phys. Rev. Lett. 122, 246401 (2019).

[41] N. Bultinck, E. Khalaf, S. Liu, S. Chatterjee, A. Vishwanath, and M. P. Zaletel, Ground State and Hidden Symmetry of Magic-Angle Graphene at Even Integer Filling, Phys. Rev. X 10, 031034 (2020).

[42] B. A. Bernevig, Z. Song, N. Regnault, and B. Lian, TBG I: Matrix Elements, Approximations, Perturbation Theory and a $k \cdot p$ 2-Band Model for Twisted Bilayer Graphene, Phys. Rev. B 103, 205411 (2021).

[43] Z.-D. Song, B. Lian, N. Regnault, and A. B. Bernevig, TBG II: Stable symmetry anomaly in twisted bilayer graphene, Phys. Rev. B 103, 205412 (2021).

[44] B. A. Bernevig, Z. Song, N. Regnault, and B. Lian, TBG III: Interacting hamiltonian and exact symmetries of twisted bilayer graphene, Phys. Rev. B 103, 205413 (2021).

[45] B. Lian, Z.-D. Song, N. Regnault, D. K. Efetov, A. Yazdani, and B. A. Bernevig, TBG IV: Exact insulator ground states and phase diagram of twisted bilayer graphene, Phys. Rev. B 103, 205414 (2021).

[46] B. A. Bernevig, B. Lian, A. Cowsik, F. Xie, N. Regnault, and Z.-D. Song, TBG V: Exact analytic many-body excitations in twisted bilayer graphene coulomb hamiltonians: Charge gap, goldstone modes and absence of cooper pairing, Phys. Rev. B 103, 205415 (2021). 
[47] F. Xie, A. Cowsik, Z. Son, B. Lian, B. A. Bernevig, and N. Regnault, TBG VI: An exact diagonalization study of twisted bilayer graphene at non-zero integer fillings, Phys. Rev. B 103, 205416 (2021).

[48] N. Bultinck, S. Chatterjee, and M. P. Zaletel, Mechanism for Anomalous Hall Ferromagnetism in Twisted Bilayer Graphene, Phys. Rev. Lett. 124, 166601 (2020).

[49] A. Abouelkomsan, Z. Liu, and E. J. Bergholtz, Particle-Hole Duality, Emergent Fermi Liquids, and Fractional Chern Insulators in Moiré Flatbands, Phys. Rev. Lett. 124, 106803 (2020).

[50] C. Repellin, Z. Dong, Y.-H. Zhang, and T. Senthil, Ferromagnetism in Narrow Bands of Moiré Superlattices, Phys. Rev. Lett. 124, 187601 (2020).

[51] C. Repellin and T. Senthil, Chern bands of twisted bilayer graphene: Fractional Chern insulators and spin phase transition, Phys. Rev. Research 2, 023238 (2020).

[52] G. Tarnopolsky, A. J. Kruchkov, and A. Vishwanath, Origin of Magic Angles in Twisted Bilayer Graphene, Phys. Rev. Lett. 122, 106405 (2019).

[53] J. Liu, J. Liu, and X. Dai, Pseudo Landau level representation of twisted bilayer graphene: Band topology and implications on the correlated insulating phase, Phys. Rev. B 99, 155415 (2019).

[54] S. Becker, M. Embree, J. Wittsten, and M. Zworski, Mathematics of magic angles in a model of twisted bilayer graphene, arXiv:2008.08489 [math-ph].

[55] P. J. Ledwith, G. Tarnopolsky, E. Khalaf, and A. Vishwanath, Fractional chern insulator states in twisted bilayer graphene: An analytical approach, Phys. Rev. Research 2, 023237 (2020).

[56] F. D. M. Haldane, A modular-invariant modified Weierstrass sigma-function as a building block for lowest-Landau-level wave functions on the torus, J. Math. Phys. 59, 071901 (2018).

[57] J. Wang, S. D. Geraedts, E. H. Rezayi, and F. D. M. Haldane, Lattice Monte Carlo for quantum Hall states on a torus, Phys. Rev. B 99, 125123 (2019).

[58] S. D. Geraedts, J. Wang, E. H. Rezayi, and F. D. M. Haldane, Berry Phase and Model Wave Function in the Half-Filled Landau Level, Phys. Rev. Lett. 121, 147202 (2018).

[59] J. Wang, Dirac Fermion Hierarchy of Composite Fermi Liquids, Phys. Rev. Lett. 122, 257203 (2019).

[60] F. D. M. Haldane and E. H. Rezayi, Periodic Laughlin-Jastrow wave functions for the fractional quantized Hall effect, Phys. Rev. B 31, 2529 (1985).

[61] R. B. Laughlin, Quantized Hall conductivity in two dimensions, Phys. Rev. B 23, 5632 (1981).

[62] D. P. Arovas, R. N. Bhatt, F. D. M. Haldane, P. B. Littlewood, and R. Rammal, Localization, Wave-Function Topology, and the Integer Quantized Hall Effect, Phys. Rev. Lett. 60, 619 (1988).

[63] M. Kohmoto, Topological invariant and the quantization of the Hall conductance, Ann. Phys. 160, 343 (1985).

[64] M. Czerwinski and E. Brown, A nodal determination of the Hall conductance, Proc.: Math. Phys. Sci. 433, 547 (1991).

[65] C. A. Swanson, Comparison and Oscillation Theory of Linear Differential Equations, edited by Richard Bellman (Elsevier, 1968).
[66] Y. Jiang, X. Lai, K. Watanabe, T. Taniguchi, K. Haule, J. Mao, and E. Y. Andrei, Charge order and broken rotational symmetry in magic-angle twisted bilayer graphene, Nature (London) 573, 91 (2019).

[67] F. Guinea and N. R. Walet, Electrostatic effects, band distortions, and superconductivity in twisted graphene bilayers, Proc. Natl. Acad. Sci. USA 115, 13174 (2018).

[68] A. A. Koulakov, M. M. Fogler, and B. I. Shklovskii, Charge Density Wave in Two-Dimensional Electron Liquid in Weak Magnetic Field, Phys. Rev. Lett. 76, 499 (1996).

[69] E. H. Rezayi and F. D. M. Haldane, Incompressible Paired Hall State, Stripe Order, and the Composite Fermion Liquid Phase in Half-Filled Landau Levels, Phys. Rev. Lett. 84, 4685 (2000).

[70] K. Yang, F. D. M. Haldane, and E. H. Rezayi, Wigner crystals in the lowest Landau level at low-filling factors, Phys. Rev. B 64, 081301(R) (2001).

[71] X. Wan and K. Yang, Striped quantum Hall state in a half-filled Landau level, Phys. Rev. B 93, 201303(R) (2016).

[72] G. Moore and N. Read, Nonabelions in the fractional quantum Hall effect, Nucl. Phys. B 360, 362 (1991).

[73] W.-Y. He, D. Goldhaber-Gordon, and K. T. Law, Giant orbital magnetoelectric effect and current-induced magnetization switching in twisted bilayer graphene, Nat. Commun. 11, 1650 (2020).

[74] T. Thonhauser, D. Ceresoli, D. Vanderbilt, and R. Resta, Orbital Magnetization in Periodic Insulators, Phys. Rev. Lett. 95 137205 (2005).

[75] D. Ceresoli, T. Thonhauser, D. Vanderbilt, and R. Resta, Orbital magnetization in crystalline solids: Multi-band insulators, Chern insulators, and metals, Phys. Rev. B 74, 024408 (2006).

[76] N. Sai, K. M. Rabe, and D. Vanderbilt, Theory of structural response to macroscopic electric fields in ferroelectric systems, Phys. Rev. B 66, 104108 (2002).

[77] D. Xiao, J. Shi, and Q. Niu, Berry Phase Correction to Electron Density of States in Solids, Phys. Rev. Lett. 95, 137204 (2005).

[78] J. Shi, G. Vignale, D. Xiao, and Q. Niu, Quantum Theory of Orbital Magnetization and its Generalization to Interacting Systems, Phys. Rev. Lett. 99, 197202 (2007).

[79] D. Xiao, M.-C. Chang, and Q. Niu, Berry phase effects on electronic properties, Rev. Mod. Phys. 82, 1959 (2010).

[80] T. Thonhauser, Theory of orbital magnetization in solids, Int. J. Mod. Phys. B 25, 1429 (2011).

[81] J. Liu, Z. Ma, J. Gao, and X. Dai, Quantum Valley Hall Effect, Orbital Magnetism, and Anomalous Hall Effect in Twisted Multilayer Graphene Systems, Phys. Rev. X 9, 031021 (2019).

[82] S.-Y. Li, Y. Zhang, Y.-N. Ren, J. Liu, X. Dai, and L. He, Experimental evidence for orbital magnetic moments generated by moiré-scale current loops in twisted bilayer graphene, Phys. Rev. B 102, 121406 (2020).

[83] H. Shi, Z. Zhan, Z. Qi et al., Large-area, periodic, and tunable intrinsic pseudo-magnetic fields in low-angle twisted bilayer graphene, Nat. Commun. 11, 371 (2020).

[84] Y. Aharonov and A. Casher, Ground state of a spin-1/2 charged particle in a two-dimensional magnetic field, Phys. Rev. A 19, 2461 (1979).

[85] P. San-Jose, J. González, and F. Guinea, Non-Abelian Gauge Potentials in Graphene Bilayers, Phys. Rev. Lett. 108, 216802 (2012). 
[86] M. J. Pacholski, C. W. J. Beenakker, and İ. Adagideli, Topologically Protected Landau Level in the Vortex Lattice of a Weyl Superconductor, Phys. Rev. Lett. 121, 037701 (2018).

[87] J. Kailasvuori, Pedestrian index theorem à la Aharonov-Casher for bulk threshold modes in corrugated multilayer graphene, Europhys. Lett. 87, 47008 (2009).
[88] Y. Ren, Q. Gao, A. H. MacDonald, and Q. Niu, WKB Estimate of Bilayer Graphene's Magic Twist Angles, Phys. Rev. Lett. 126, 016404 (2021).

[89] F. K. Popov and A. Milekhin, Hidden wave function of twisted bilayer graphene: Flat band as a Landau level, Phys. Rev. B 103, 155150 (2021). 